\title{
Statics and Dynamics of Malaria Transmission: The Relationship between Prevalence in Humans and Mosquitoes.
}

Santiago Movilla Blanco ( $\sim$ sbl042@uib.no)

University of Bergen, Bergen, Norway.

\section{Methodology}

Keywords: malaria epidemiology, mosquitoes , malaria parasites, vector species

Posted Date: September 18th, 2020

DOI: https://doi.org/10.21203/rs.3.rs-73072/v1

License: (c) (1) This work is licensed under a Creative Commons Attribution 4.0 International License.

Read Full License 


\title{
Statics and Dynamics of Malaria Transmission: The Relationship between Prevalence in Humans and Mosquitoes.
}

\author{
Santiago Movilla Blanco. sbl042@uib.no
}

System Dynamics Group, Department of Geography, University of Bergen, Bergen, Norway.

\section{Abstract.}

The present paper explores a simple dynamic model from which we review the classic formulae in malaria epidemiology that relate entomological parameters to malaria transmission. In addition, we document the dynamics of malaria, illustrating the impact of control strategies and how the bites per mosquito have a larger effect on transmission intensity than the mosquito mortality, the ratio of mosquitoes to humans, or the transmission efficiency.

The model has been built following the System Dynamics methodology, explicitly representing the variables, the feedbacks and the nonlinearities, i.e. the structure that governs the dynamics of the disease. In this sense, the paper offers a new way to obtain the most representative malaria indicators derived from stock-and-flow diagrams that encompass the causal relationships that exist between the attributes of such a system.

Based on the obtained formulae from the human and mosquito sectors, we are able to eliminate three degrees of freedom, allowing us to calculate the temporal steady state relationship between Plasmodium falciparum prevalence in humans and mosquitoes.

The model is generic in nature and may be parameterized to portray a wide variety of locations, different malaria parasites, vector species, and to cater for seasonality.

Given that the model includes the principle mechanisms of malaria transmission, it acts as a foundation for simulations that represent the dynamics between humans and mosquitoes. Such model has been developed based on a number of simplifying assumptions. To the extent possible, the validity of the model under these assumptions has been analyzed by way of mathematic equations.

\section{Introduction.}

Malaria in humans is caused by 5 Plasmodium parasites: Plasmodium falciparum, P. vivax, P. malariae, P. ovale and P. knowlesi [1] https. Among all the species, P. falciparum is the most prevalent and dangerous malaria parasite. Only in Africa, where most of the malaria is concentrated, over $95 \%$ of episodes are caused by P. falciparum [2] https.

Due to its impact worldwide, we have chosen to focus our analysis on the transmission of P. falciparum. The analysis would be similar, though not the same, for other malaria parasites. For example, infections by the variant, P. vivax are characterized by relapses of malaria arising from the persistent liver stage of 
the parasite (hypnozoites) [3] https, which results in a different model structure than the case of P. falciparum.

In order to understand the epidemiology of malaria and other infectious diseases, mathematical models have historically played an important role. Models provide concise quantitative descriptions of complicated, non-linear processes, and a method for relating the process of infection in individuals to the incidence of infection or disease in a population over time [4] https.

The System Dynamics (SD) modeling methodology is well suited to address the dynamic complexity that characterizes many public health issues. This approach involves the development of computer simulation models that portray processes of accumulation and feedbacks and that may be tested systematically to find effective policies for overcoming policy resistance [5] https.

In line with health issues, mosquito control is one of the key components in the fight against malaria. To ensure an effective implementation of interventions to reduce malaria transmission, the SD methodology is suitable to develop decision-support tools. These tools can help policy makers in improving the design of strategies that maximize reduction in mosquito bites and minimize the costs of malaria reduction, greatly impacting on malaria transmission at local and national level.

Given the simplicity and the purpose of this model, its comprehensiveness and extensiveness are limited. In this paper it only portrays epidemiological aspects of malaria transmission. However, the model can include a higher level of complexity. In addition, the model is easily adaptable and can be customized for any location where P. falciparum is prevalent.

The structure is inspired by the Ross-Macdonald model of malaria transmission (Figure 01) [6] https, and it is divided in two sectors.

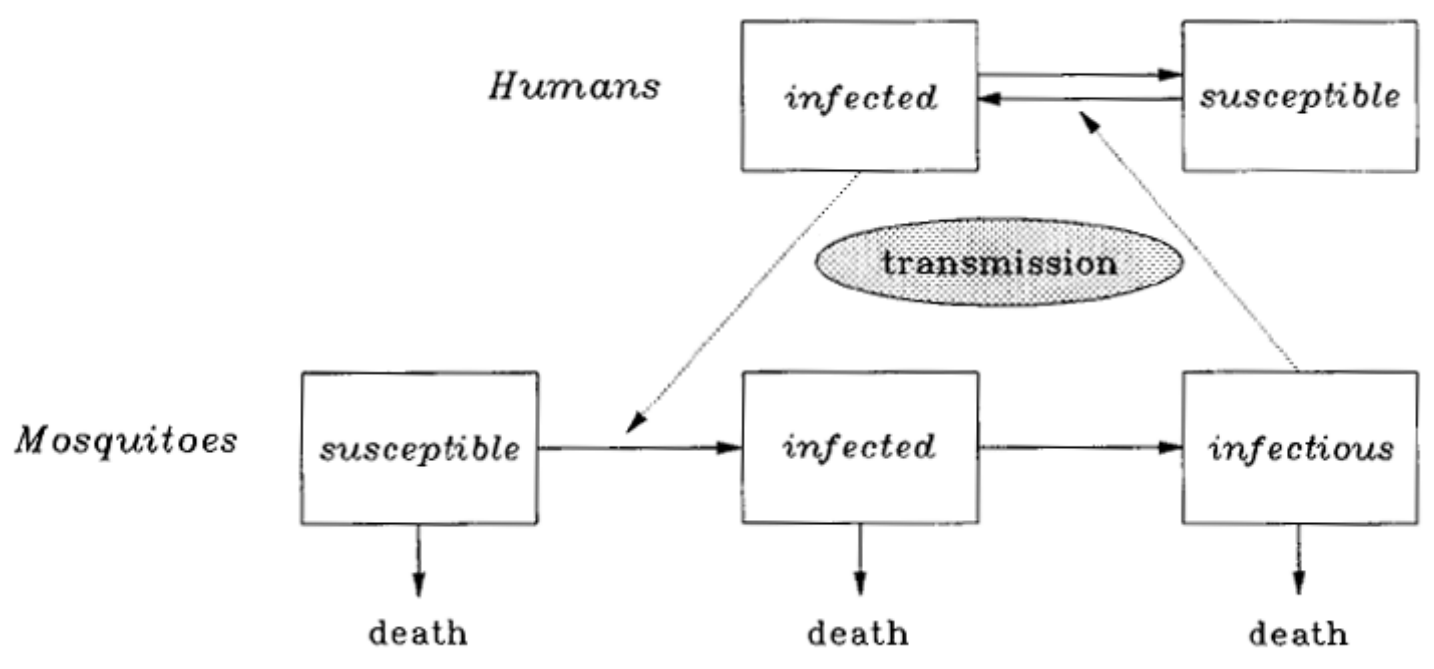

Figure 01 
The first sector analyzes the humans and the second the mosquitoes. The common factor between the two sectors is humans and mosquitoes carrying the malaria parasite, i.e. P. falciparum. In this paper we will examine the simplest version of the model but covering the major aspects of malaria transmission.

\section{Human Sector.}

The human sector portrays the dynamics of the different human states regarding malaria. We will first define the different indicators involved, and later we will analyze the connection between them.

The figure 02 contains a list of indicators with the abbreviations that we will use for the equations.

$$
\begin{aligned}
& H=\text { Human Population } \\
& H_{S}=\text { Susceptible Humans } \\
& H_{I}=\text { Infected Humans } \\
& P_{H}=\text { Human Prevalence } \\
& P_{M}=\text { Mosquito Prevalence } \text { or Sporozoite Rate } \\
& V_{R}=\text { Vector Biting Rate } \\
& \text { EIR = Entomological Inoculation Rate } \\
& I_{M}=\text { Mosquito Infectivity } \\
& T_{R}=\text { Average Recovery Time }
\end{aligned}
$$

Figure 02

Some indicators are named in a simplified way but we will describe one by one.

- Human Population (H): This refers to the total number of humans living in the area of analysis.

- Susceptible Humans $\left(\mathbf{H}_{\mathbf{s}}\right)$ : We first define susceptible humans as persons clean from the malaria parasite and in consequence susceptible to get infected with malaria if bitten by an infectious Anopheles mosquito.

- Infected Humans $\left(\mathbf{H}_{1}\right)$ : According to the WHO malaria terminology [7] https, malaria infected people are those with the presence of Plasmodium parasites in blood or tissues confirmed by diagnostic testing. Therefore, infected humans are the number of persons carrying P. falciparum parasites at a given point in time.

- Human Prevalence $\left(\mathbf{P}_{\mathrm{H}}\right)$ : In epidemiology the prevalence of a certain disease is often defined as the number of individuals with infection at one time, but in our case we will define the prevalence in terms of proportion. Human prevalence is therefore defined as the population fraction that has parasitemia and it is obtained by dividing the infected humans $H_{1}$ by the total number of people $H$.

- Mosquito Prevalence $\left(\mathbf{P}_{\mathrm{M}}\right)$ : Similarly to how we defined human prevalence, the mosquito prevalence is also a proportion, in this case, the proportion of infectious mosquitoes. This indicator is also known as Sporozoite Rate or in our case P. Falciparum Sporozoite Rate, which is the number of mosquitoes infected with sporozoites (the life parasite stage responsible for transmission from mosquito to human), divided by the total number of mosquitoes [8] https. 
In practice, the determination of sporozoite rates are obtained as the percentage of $P$. falciparum sporozoite positive mosquito samples out of the total number of mosquitoes analyzed [9] https.

- Vector Biting Rate $\left(\mathbf{V}_{\mathbf{R}}\right)$ : This indicator is also known as the Human Biting Rate of the Anopheles mosquitoes, and it is calculated as the total number of indoor and outdoor mosquitoes caught biting humans during a 12-month sampling period divided by the number of people bitten (the catchers) [9].

Although the value of this indicator is obtained through the number of mosquitoes caught biting humans, these mosquitoes also represent the number of bites received by a person during one year, and we can use the equivalent units of mosquito bites per person per year (bites/(person-year)). If the sampling period is not one year, we multiply the number of bites by the corresponding factor to make it annual. For example, if the bites are collected during a sampling period of four months, we will multiply the bites per person by three in order to get the bites in per annum units. It is important to note that this multiplication is only meant to adapt the units since the obtained Vector Biting Rate $\left(V_{R}\right)$ can only be used for the period during which it is valid. In fact, values obtained during wet seasons are likely to be higher than during dry seasons given that the rain normally involves increases in mosquito density.

-Entomological Inoculation Rate (EIR): The intensity of malaria parasite transmission is normally expressed as the entomological inoculation rate (EIR), the product of the vector biting rate times the proportion of mosquitoes infected with sporozoite-stage malaria parasites [10] https.

EIR is similarly defined as a measure of exposure to infectious mosquitoes. It is usually interpreted as the number of $P$. falciparum infective bites received by an individual during a season or in our case, annually [8]. Therefore, we refer to the annual entomological inoculation rate (AEIR), which is obtained by multiplying the average annual vector biting rate by the mosquito prevalence [9].

The equation for the EIR is indicated in (01) as the Vector Biting Rate $\left(V_{R}\right)$ multiplied by the Mosquito Prevalence $\left(\mathrm{P}_{\mathrm{M}}\right)$ :

$$
E I R=V_{R} \cdot P_{M}
$$

The units for this indicator are also bites per person per year, but the EIR refers only to the number of infectious bites. If we had a number for all the annual infectious bites in the area of analysis, the EIR would be the infectious bites per capita.

-Mosquito Infectivity $\left(I_{M}\right)$ : Also known as transmission efficiency from mosquito to human, this indicator is defined as the probability of an infectious mosquito to infect a susceptible human during blood-feeding. Normally, when a mosquito carrying sporozoites bites a susceptible human, there is $100 \%$ chance that the parasites will be passed to the individual. However, whether the host becomes infected or not depends on other factors that might reduce mosquito infectivity. For example, it has been long assumed that the probability of mosquito-to-human transmission is determined simply by the presence of salivary gland sporozoites. However, the number of parasites injected might influence the chances of disease transmission [11] https. 
In summary, assuming a bite from an infectious mosquito, we can define mosquito infectivity as the probability to inoculate sporozoites that invade the liver cells of the host, and initiate the infection process.

The unit for this indicator is fractional number of persons infected per bite. Its maximum value would be 1 , where every bite from an infectious mosquito on a susceptible human would constitute a human infection. In such case the incidence or number of malaria infections per year would be the EIR multiplied by the number of susceptible humans.

-Average Recovery Time $\left(T_{R}\right)$ : This is the average time between human infection and clearance of parasites in the blood. In our model an infected person becomes susceptible again after this time. If the person has developed immunity to become infected again, this will be reflected in the reduction of the mosquito infectivity.

This indicator is calculated as the weighted average between the recovery time for persons who feel the malaria symptoms and recover shortly after treatment, and the recovery time for asymptomatic infections, which normally do not get treated and prolong the infection for significant periods of time.

In general, it takes around two weeks of treatment to recover from malaria. But without clinical illness, asymptomatic infections are silent and remain untreated, resulting in chronic carriage that can last for 6 months or longer [12] https. In this sense, a study conducted in an area of high P. falciparum transmission in Ghana estimated that untreated asymptomatic infections had a mean duration of 194 days ( 0.53 years) [13] https.

We will elaborate more the calculation of this indicator later as the complexity of the model increases. In order to keep consistency with the model, the time units for recovery time are in years.

Once defined the first list of indicators, we can see them linked in the model diagram (Figure 03). Stocks are represented with rectangles while the thick arrows represent the flows. The thin arrows represent effects or causal relationships between two variables. The polarities next to the thin arrows (plus or minus) indicate whether the effect is positive or negative. For example, a bigger EIR increases the incidence (positive effect), but at the same time, a bigger recovery time reduces the recovering flow (negative effect). 


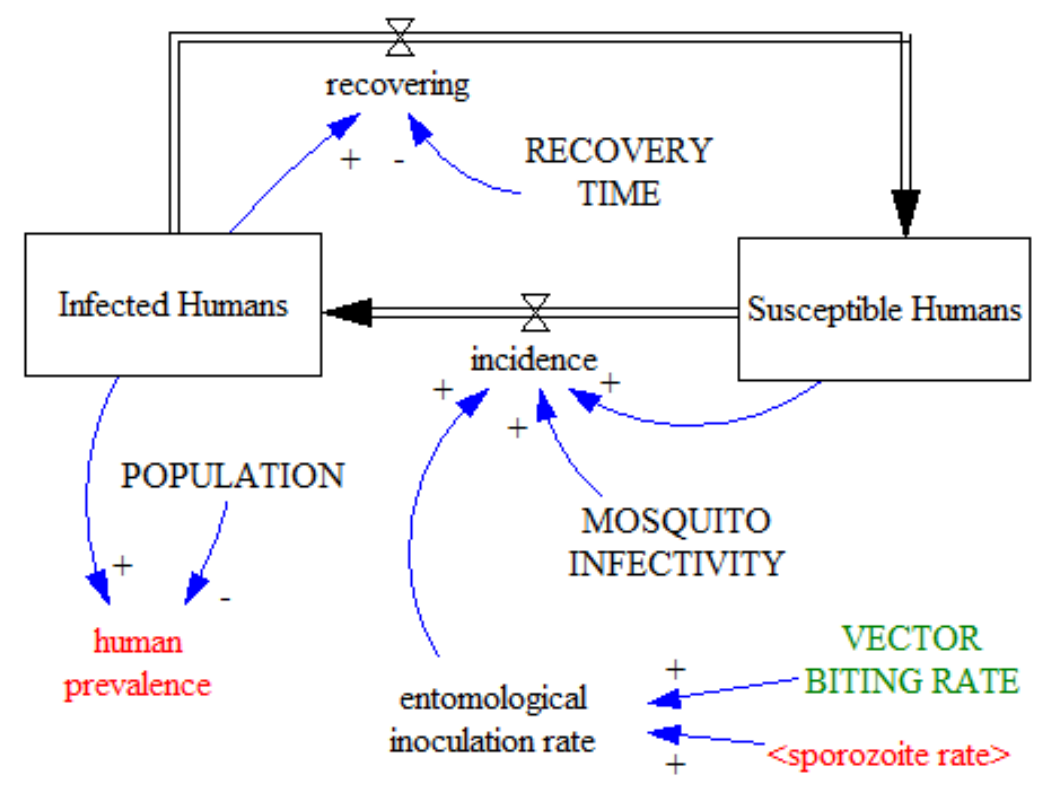

Figure 03

As in the Ross-Macdonald model, there are two stocks, one with the susceptible population and the other with infected population. People go from susceptible humans to infected humans through the flow of incidence, which is defined as the number of newly diagnosed malaria cases during a defined period. In our case the period is one year and we consider annual infections with units in persons/year.

The persons in the stock of infected humans recover eventually through the flow of recovering, also in persons/year, going from infected back to susceptible. The recovering is determined by the two elements from which it gets linking arrows. In this case recovering is defined as the stock on infected humans divided by the average recovery time.

In an equilibrium situation, the number of infections and recovering are similar in order to keep the stock values relatively constant over time. This means we could assume both flows with same values over time (incidence $=$ recovering).

We also know that the human prevalence $\left(P_{H}\right)$ is calculated as a fraction, dividing the stock of infected humans by the total population as explained previously. However, if we refer to human prevalence as the number of individuals with infection at one time, then human prevalence is equal to the stock of infected humans.

In this case, recovering would be equal to prevalence divided by recovery time, and since in equilibrium recovering is equal to incidence, then incidence would be also equal to prevalence divided by recovery time. As a result, prevalence is the incidence multiplied by the recovery time.

Prevalence $=$ Incidence $\cdot$ Recovery Time. This is the formula generally used in epidemiology and we can see that it fits with the structure of our preliminary model.

The rest of equations associated to the model structure in figure 02 are determined as follows: 
If human prevalence $\left(P_{H}\right)$ is equal to Infected Humans $\left(H_{1}\right)$ divided by all humans $(H)$, then infected humans is prevalence multiplied by total humans. We can also obtain the susceptible population $\left(H_{s}\right)$ as the total humans minus the infected.

$$
\begin{aligned}
& H_{I}=H \cdot P_{H} \\
& H_{S}=H-H \cdot P_{H}=H \cdot\left(1-P_{H}\right)
\end{aligned}
$$

As indicated before, the flow of recovering is equal to the stock of infected humans divided by average recovery time. The flow of incidence or annual infections is equal to the susceptible population multiplied by the annual infectious bites per person (EIR), and multiplied by the mosquito Infectivity $\left(I_{M}\right)$. Substituting from $(02)$ we get the following equations:

Recovering $=\frac{H_{I}}{T_{R}}=\frac{H \cdot P_{H}}{T_{R}}$

Incidence $=H_{S} \cdot E I R \cdot I_{M}=H \cdot\left(1-P_{H}\right) \cdot E I R \cdot I_{M}$

Considering the system is in equilibrium, incidence must be equal to recovering. Substituting from (03) we get the following equations:

$$
\begin{aligned}
& \text { Recovering }=\text { Incidence } \rightarrow \frac{H \cdot P_{H}}{T_{R}}=H \cdot\left(1-P_{H}\right) \cdot E I R \cdot I_{M} \Rightarrow \frac{P_{H}}{T_{R}}=\left(1-P_{H}\right) \cdot E I R \cdot I_{M} \Rightarrow \\
& \Rightarrow P_{H}=\left(1-P_{H}\right) \cdot E I R \cdot I_{M} \cdot T_{R} \Rightarrow P_{H}+P_{H} \cdot E I R \cdot I_{M} \cdot T_{R}=E I R \cdot I_{M} \cdot T_{R} \Rightarrow\left[P_{H}=\frac{E I R \cdot I_{M} \cdot T_{R}}{1+E I R \cdot I_{M} \cdot T_{R}}\right]
\end{aligned}
$$

The resulting equation in (04) relates human prevalence with the EIR, depending on the value of recovery time and mosquito infectivity. This relationship between EIR and the proportion of humans with parasitemia can be seen in the next graph (Figure 04), where we have assumed $I_{M}=1$ person/bite and $\mathrm{a} \mathrm{T}_{\mathrm{R}}=0.25$ years, i.e. 3 months.

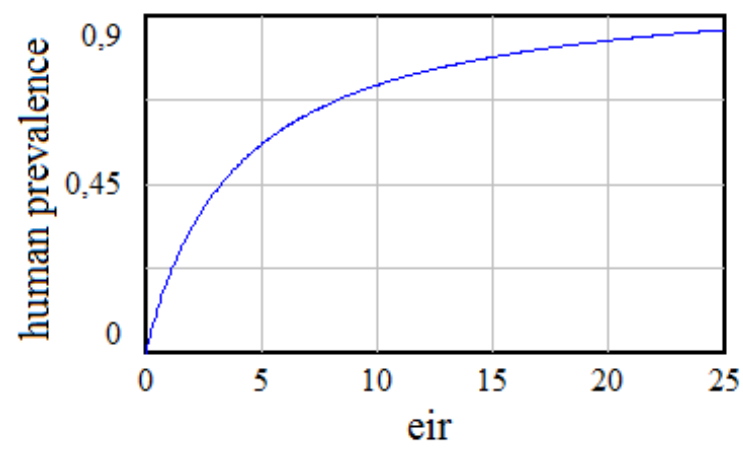

Figure 04

The shape of this graph is the same as the obtained in other mathematical studies [4], and also matches with other graphs obtained empirically [14] https. 
If we substitute the equation (01) that calculates the EIR, in the equation (04) that obtains the human prevalence $\mathrm{P}_{\mathrm{H}}$, we obtain the following expression:

$\left.\begin{array}{l}P_{H}=\frac{E I R \cdot I_{M} \cdot T_{R}}{1+E I R \cdot I_{M} \cdot T_{R}} \\ E I R=P_{M} \cdot V_{R}\end{array}\right\} \Rightarrow\left[P_{H}=\frac{P_{M} \cdot V_{R} \cdot I_{M} \cdot T_{R}}{1+P_{M} \cdot V_{R} \cdot I_{M} \cdot T_{R}}\right]$

From this formula we can also deduce the value of the vector biting rate $V_{R}$, assuming that we know the rest of the indicators:

$$
\begin{gathered}
P_{H}=\frac{P_{M} \cdot V_{R} \cdot I_{M} \cdot T_{R}}{1+P_{M} \cdot V_{R} \cdot I_{M} \cdot T_{R}} \Rightarrow P_{H}+P_{H} \cdot P_{M} \cdot V_{R} \cdot I_{M} \cdot T_{R}=P_{M} \cdot V_{R} \cdot I_{M} \cdot T_{R} \Rightarrow \\
\Rightarrow P_{H}=P_{M} \cdot V_{R} \cdot I_{M} \cdot T_{R} \cdot\left(1-P_{H}\right) \Rightarrow\left[V_{R}=\frac{P_{H}}{P_{M} \cdot I_{M} \cdot T_{R} \cdot\left(1-P_{H}\right)}\right]
\end{gathered}
$$

For simplicity, and given that we consider a steady state model, we can ignore births and deaths as the total population does not experience major changes in a temporal equilibrium system. In fact, the assumption that a population is at dynamic equilibrium and has a stationary age distribution is frequently violated in practice, but it provides a useful starting point for more sophisticated analysis [4].

In more complex versions of the model we have included human births and deaths, and even after implementing a dynamic population that varies over time, the final equations that link human and mosquito prevalence still provide very similar results to the ones we just obtained. This similitude is principally supported by the part of the model that deals with the mosquitoes, which operates with units in mosquitoes per person, and consequently produces very similar results regardless of the changing number of persons.

\section{Mosquito Sector.}

As we did in the human sector, we will first define the different indicators involved, and later we will analyze the connection between them.

The figure 05 contains a list of indicators with the abbreviations that we will use for the equations.

$$
\begin{aligned}
& B_{R}=\text { Annual Bites per Mosquito } \\
& M=\text { Biting Mosquitoes per Person } \\
& M_{I}=\text { Infectious Mosquitoes per Person } \\
& M_{S}=\text { Susceptible Mosquitoes per Person } \\
& T_{P}=\text { Time to Develop Sporozoites } \\
& T_{M}=\text { Longevity Adult Mosquito } \\
& T_{E}=\text { Longevity Infected Mosquito } \\
& T_{I}=\text { Longevity Infectious Mosquito } \\
& I_{H}=\text { Human Infectivity } \\
& I_{R}=\text { Mosquito Infectious Rate }
\end{aligned}
$$


Figure 05

-Annual Bites per Mosquito $\left(B_{R}\right)$ : Also known as human feeding rate, this indicator represents the average number of bites on humans per mosquito and per year. Anopheles mosquitoes can also take their blood meals from other animals but those bites do not count as part of our annual bites per mosquito. Therefore, among other factors, this value also depends on the human blood index (HBI), which is defined as the proportion of mosquito blood meals from humans in the area of study [7].

If we consider the longevity of adult female mosquitoes around three weeks [15] https, the annual bites would be equal to the bites by a mosquito during its lifetime multiplied by the number of three-week periods in one year. Therefore, the annual bites do not depend on the mosquito longevity and represents the level of human biting activity during the mosquito lifetime. The units are in bites/(mosquito·year).

This indicator is only applicable to adult female Anopheles mosquitoes, which are the blood feeding mosquitoes responsible for malaria transmission. Depending on the conditions, female mosquitoes would normally search for a blood meal every 2-3 days [16] https, resulting in around 150 bites per year. However, these bites are distributed among different animal species and the number of bites on humans is inferior. In our case the bites per mosquito are calculated mathematically in the model taking as a reference other indicators obtained empirically.

-Biting Mosquitoes per Person (M): This variable represents a way to quantify the mosquito density in the area of analysis. There are various methods as replacement of human landing catches (HLC) for mosquito sampling [17] https. However, in our case the biting mosquitoes per person are obtained mathematically from the Vector Biting Rate $\left(V_{R}\right)$, which we already defined as the annual number of mosquitoes caught biting humans divided by the number of people bitten, with units in bites/(person·year).

If we were able to multiply the bites per mosquito $\left(B_{R}\right)$ by all the biting mosquitoes per person $(M)$, it would result in the total number of bites received by one individual, which is precisely our Vector Biting Rate. Therefore, as indicated in (07), the number of biting mosquitoes per person is the result from dividing all the bites per person $\left(V_{R}\right)$ by the bites per mosquito $\left(B_{R}\right)$.

$V_{R}=B_{R} \cdot M \Rightarrow M=\frac{V_{R}}{B_{R}}$

Out of the three indicators included in (07), we consider the Vector Biting Rate $\left(V_{R}\right)$ as the only known indicator by observation. The female Anopheles mosquitoes that have never bitten humans are not recorded through the Vector Biting Rate $\left(V_{R}\right)$, but they are indirectly recorded by the mosquito prevalence $\left(P_{M}\right)$, and they are also part of the Biting Mosquitoes per Person (M) because they contribute reducing the average bites per mosquito $\left(B_{R}\right)$.

Later we will calculate the Annual Bites per Mosquito $\left(B_{R}\right)$ and the Biting Mosquitoes per Person (M) during the explanation of the mosquito sector. 
-Infectious Mosquitoes per Person ( $\left.\mathbf{M}_{1}\right)$ : Out of the total mosquitoes per person (M), the infectious mosquitoes are those that are able to transmit malaria. We can obtain this number multiplying mosquitoes per person $(M)$ by the mosquito prevalence $\left(P_{M}\right)$, as indicated in (08).

$M_{I}=M \cdot P_{M}$

-Susceptible Mosquitoes per Person $\left(\mathrm{M}_{\mathbf{s}}\right)$ : For the moment we will assume that susceptible mosquitoes are the total mosquitoes $(M)$ minus the infectious $\left(M_{1}\right)$. Using the result in (08) we determine $M_{s}$ by following the next equation:

$M_{S}=M-M_{I}=M-M \cdot P_{M}=M \cdot\left(1-P_{M}\right)$

We know that mosquitoes develop infectiousness by going through a process from susceptible to infectious. During that process the mosquitoes are in an intermediate state called infected mosquitoes, where they are neither infectious nor susceptible. Therefore, during the process of becoming infectious they remain infected until they die or until they actually become infectious. Later we will include a more complex model with infected mosquitoes and explain why this preliminary assumption is valid for our analysis, but for now we will assume that the infected mosquitoes are part of the stock of susceptible mosquitoes.

-Time to Develop Sporozoites $\left(T_{P}\right)$ : Assuming a mosquito becoming infectious, this indicator represents the period between the bite to a human carrying gametocytes (the life parasite stage that do not cause disease symptoms but is responsible for onward transmission to mosquitoes), and the presence of sporozoites in the salivary glands of the mosquito. After this time the mosquito becomes infectious and ready to transmit malaria.

$T_{p}$ is also known as the entomological incubation period, defined as the length of the incubation period, from ingestion of gametocytes to becoming infectious [4].

Its value depends on many factors but most sporozoite infections do not appear until 10 or more days after blood-feeding [18] https.

-Longevity of Adult Mosquito $\left(\mathbf{T}_{M}\right)$ : As mentioned before, the longevity of the adult female Anopheles is around three weeks [15]. This is the period during which the mosquitoes are able to bite humans.

-Longevity of Infected Mosquito $\left(T_{\mathrm{E}}\right)$ : We will use this indicator after introducing the model that includes the stock of infected mosquitoes. This is the average lifetime of a mosquito that already initiated the infection process.

The mosquitoes seek for blood meals very early after becoming adults. Immediately following emergence, there is period of one or two days during which host-seeking behavior first begins to be expressed [19] https. In this sense, the bite that triggers the infection of a mosquito could happen almost any moment during its entire adult life. Some mosquitoes could have it in their early days and others at the very end of their lifespan. 
If a susceptible mosquito performs the infective bite at the beginning of its adult life, the longevity will be close to the full longevity of an adult mosquito i.e. three weeks. On the other hand, if the infective bite is produced right before it dies, the longevity would be something close to zero. This is the reason why we think that an appropriate value for the longevity of an infected mosquito would be equal to the longevity of the adult mosquito $\left(T_{M}\right)$ divided by two, which is the average $\left(T_{E}=T_{M} / 2\right)$. The value would be something around 10 days.

-Longevity of Infectious Mosquito $\left(T_{1}\right)$ : This is the average lifetime of a mosquito that already became infectious.

Considering the longevity of an adult mosquito $\left(T_{M}\right)$ and the time to develop sporozoites $\left(T_{P}\right)$, the maximum longevity of an infectious mosquito would take place when the infective bite occurs at the earliest days of its adult life. In this case the mosquito would become infectious after $T_{p}$, and during the rest of its longevity it would remain infected. Other mosquitoes would become infectious during the last living days or never live long enough to become infectious.

Following the same logic as for the previous indicator, we have a maximum longevity and a minimum equal to zero. Therefore, the longevity of an infectious mosquito would be the middle point, obtained following the next equation.

$T_{I}=\frac{T_{M}-T_{P}}{2}$

Depending of the conditions this would result in an average period of 3 to 5 days. Given the feeding frequency, a mosquito would infect one or two persons at most unless the blood meals are interrupted. If the bites are interrupted the mosquito could infect more people.

Since all the time units in the model are years, we need to convert the longevity values calculated in days into years. In order to do that, we divide the longevities by a factor equal to 365 days/year.

-Human Infectivity $\left(I_{H}\right)$ : Similarly as we defined mosquito infectivity in the human sector (probability of an infectious mosquito to infect a susceptible human during blood-feeding), we define human infectivity as the probability of a susceptible mosquito to pick up infection from an infected human during bloodfeeding. In other words, this is the proportion of blood meals taken on infected humans which are infectious to mosquitoes. Therefore, its value ranges from 0 to 1 and the unit for this indicator is fractional number of mosquitoes infected per bite.

The obtaining of this indicator is complex: To begin with, from all the infected people that comprise the human prevalence, we need to consider only those who are infectious. But even considering only infectious persons, there is no certainty yet that susceptible mosquitoes become infected after biting these people. A study in New Guinea determined that the mean percentage of mosquitoes which became infected by feeding on infectious people was $37.9 \%$ [20] https.

Given that from the human sector we only import human prevalence, we need to consider which part of the prevalence corresponds to infectious humans as part of the calculation of the human infectivity. 
In our case, infectious humans are those carrying mature P. falciparum gametocytes in the peripheral blood and in consequence making them available for uptake by a biting Anopheles mosquito [21] https.

In this sense, gametocyte density might not be related to infectiousness. In one study they found some individuals with low gametocyte densities who were infective to mosquitoes, while others with abundant gametocytes did not infect [22] https. This result is from an old study and perhaps they did not take the maturity of the gametocytes into consideration. Consequently, it is important to keep in mind that not all gametocyte carriers are infectious, but only those with mature gametocytes in the peripheral blood.

In summary, the concept of malaria infectious and malaria infected are not the same. Malaria infectious implies carrying mature gametocytes while the definition of malaria infection just implies the presence of parasites confirmed by diagnostic testing, without specifying whether parasitemia is asexual (merozoites), sexual (gametocytes), or both. And as we indicated, not even all the identified gametocyte carriers are infectious. Therefore, we can make the assumption that all the individuals included in the malaria prevalence are potentially infectious, and human infectivity will be equal to the proportion of mosquitoes that pick up infection after biting a member of the human prevalence i.e. infected person.

With the given premises, our human infectivity can be obtained through the multiplication of three factors: 1. Proportion of the prevalence carrying gametocytes, 2. Proportion of gametocyte carriers who are infectious, and 3. Proportion of susceptible mosquitoes becoming infected after biting an infectious person (or proportion of bites on infectious persons that triggers mosquito infection).

-Mosquito Infectious Rate $\left(I_{R}\right)$ : Also known as transmission efficiency from human to mosquito, this last indicator is related to the human infectivity that we just defined. If human infectivity is the probability of a susceptible mosquito to pick up infection from an infected human during blood-feeding, the mosquito infectious rate is the probability of a susceptible mosquito to become infectious after biting an infected human.

As in the human infectivity, the units are in mosquitoes/bite. But the difference between the two indicators is that human infectivity refers to the fraction of mosquitoes becoming infected and a mosquito infectious rate refers to the fraction of mosquitoes becoming infectious.

Since the mosquitoes take some time to become infectious after picking up infection, many infected mosquitoes die during the process, making the infectious rate $\left(I_{R}\right)$ significantly smaller than the human infectivity $\left(I_{H}\right)$.

The reason to use $I_{R}$ is that in this simple version of the model we do not include the stock of infected mosquitoes so we connect directly susceptible with infectious through a flow of mosquitoes which is regulated by the infectious rate as seen in figure 06. 


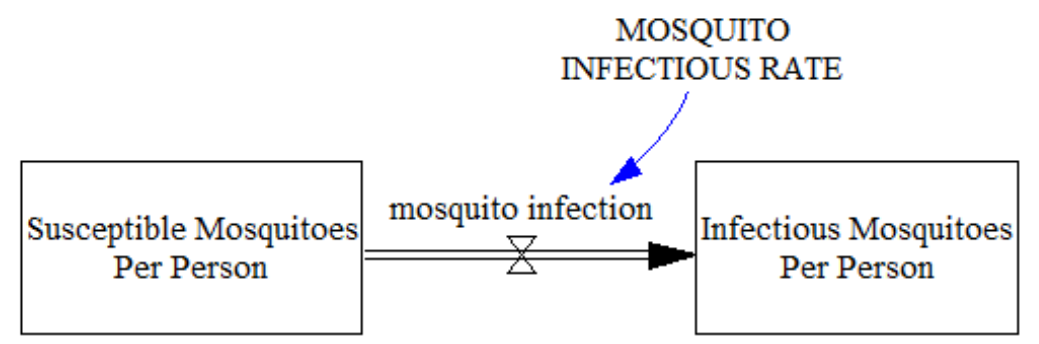

Figure 06

As a result, the susceptible mosquitoes remain in the stock of susceptible until they have plasmodium sporozoites in the salivary glands ready to inoculate on humans, making them infectious.

Later in the paper we well calculate mathematically the relationship between the Mosquito Infectious Rate and the Human Infectivity and demonstrate that this simple model gets similar results to the one including the intermediate stock of infected mosquitoes, or mosquitoes developing infectiousness.

But before we proceed with this sector we need to add some considerations:

So far we have assumed that the infectious persons are part of the reported malaria positives, and consequently being also part of the prevalence. However, it is possible that some infectious people are not recorded as part of the prevalence because the given diagnostic testing did not detect parasitemia.

These infectious individuals can be among the partially immune humans who have gametocytes present in the blood and could be infectious to mosquitoes, yet their parasites are undetected by the given diagnostic testing. There is actually evidence that chronic subpatent infections are a source of onward mosquito transmission of malaria. In semi-immune individuals with persistent infections, gametocytaemia at densities below the limit of microscopy detection is often infectious [23] https.

In this regard, one of the factors that determine parasite detection is the sensitivity of the diagnostic testing. High sensitivity tests can detect low parasite densities, but in most cases the prime concern of the detection methods is to identify asexual parasitemia, which is what causes the malaria symptoms and for which most treatments are deployed.

The most spread identification method for malaria in Africa is the rapid diagnostic test (RDT) [24] https. Interestingly, while the aim is to detect the asexual parasites that cause the disease, RDTs can lead to positives with the mere presence of gametocytes in blood (we consider the most typical RDTs that test for either or both types of malaria antigens: histidine-rich protein II (HRP2); and Plasmodium lactate dehydrogenase (pLDH) enzyme) [25] https.

Detecting possible gametocyte carriers can be useful to identify potential infectious population, but when the result from a RDT only indicates positive or negative, the positive cannot establish whether detected parasitemia is asexual, sexual or both, and in consequence positives from this method can lead to wrong diagnosis and treatment. 
For example, artemisinin-based combination treatments (ACTs) are now generally accepted as the best treatments for uncomplicated falciparum malaria [26] https. Apart from eliminating the merozoites, ACTs for falciparum malaria also reduce gametocyte carriage, and therefore reduce transmission, but artemisinin derivatives only acts against young gametocytes [27] http. Mature gametocytes are not affected by many antimalarial drugs and may thus be present long after asexual parasitemia has been cleared and the patient has recovered from the malaria symptoms [28] https.

If the RDTs used in the area of study detect mature gametocytes, we could assume that most potential infectious are within the measured prevalence. Should this assumption compromise significantly the accuracy of the results, we will use a factor that corrects the indicators before implementing values into the model.

The last consideration is about clarifying our definition of the recovery time $\left(T_{R}\right)$ in the human sector. As indicated before, $T_{R}$ is the average time between human infection and clearance of parasites in the blood. Consequently, the persons who recover from clinical symptoms after treatment but remain infectious are not considered susceptible. Therefore, our recovery period also includes the time that takes a person to clear mature gametocytes from peripheral blood circulation.

One trial demonstrated that gametocytes persist an average of 55 days after treatment with a non-ACT and 13.4 days after treatment with an ACT [29] https. These findings confirm that gametocytes may linger in peripheral blood up to several weeks after an asexual parasite infection has been cleared (whether by natural immunity or by drugs) [12].

The fact that our recovery time also includes gametocyte clearance is one of the reasons why we have not incorporated a stock with immune humans in this simple model. In general, it is important to incorporate immunity into malaria models in order to make them more realistic [6], but in our case we can incorporate them as part of the susceptible humans or as part of the infected, depending on the acquired immunity:

As mentioned before, in case of immune persons who are able to inhibit sporozoite inoculation and remain clean of parasites, we can include them in the stock of susceptible and consider their contribution in reducing the average mosquito infectivity. Although in general, people with naturally acquired immunity (NAI) still have parasitemia and we include them as infected.

Sometimes, individuals that have been permanently exposed to the parasite develop specific immune responses capable of blocking parasite transmission to the mosquito vector. This is referred to as transmission blocking immunity [30] http. However, NAl does not appear to inhibit gametocytogenesis, since immune adults retain the capacity to infect mosquitoes and constitute a sizeable and important part of the available gametocyte reservoir [31] https.

In this sense, asymptomatic infections may be associated with a greater probability of gametocyte carriage, although there is also likely to be a lower density of gametocytes in these individuals, and these effects may cancel themselves out with respect to altering the infectivity of asymptomatic infections compared to symptomatic. [12] 
But even in areas of low transmission, the contribution of asymptomatic infections to transmission is likely to be substantial, and in areas with seasonal transmission, asymptomatic infections may serve as the source of infections for a new generation of mosquitoes emerging after the start of the rains.

\subsection{Simple Mosquito Sector.}

After dealing with the previous considerations, and once defined all the indicators of the mosquito sector, we can see them linked in the next model diagram (Figure 07).

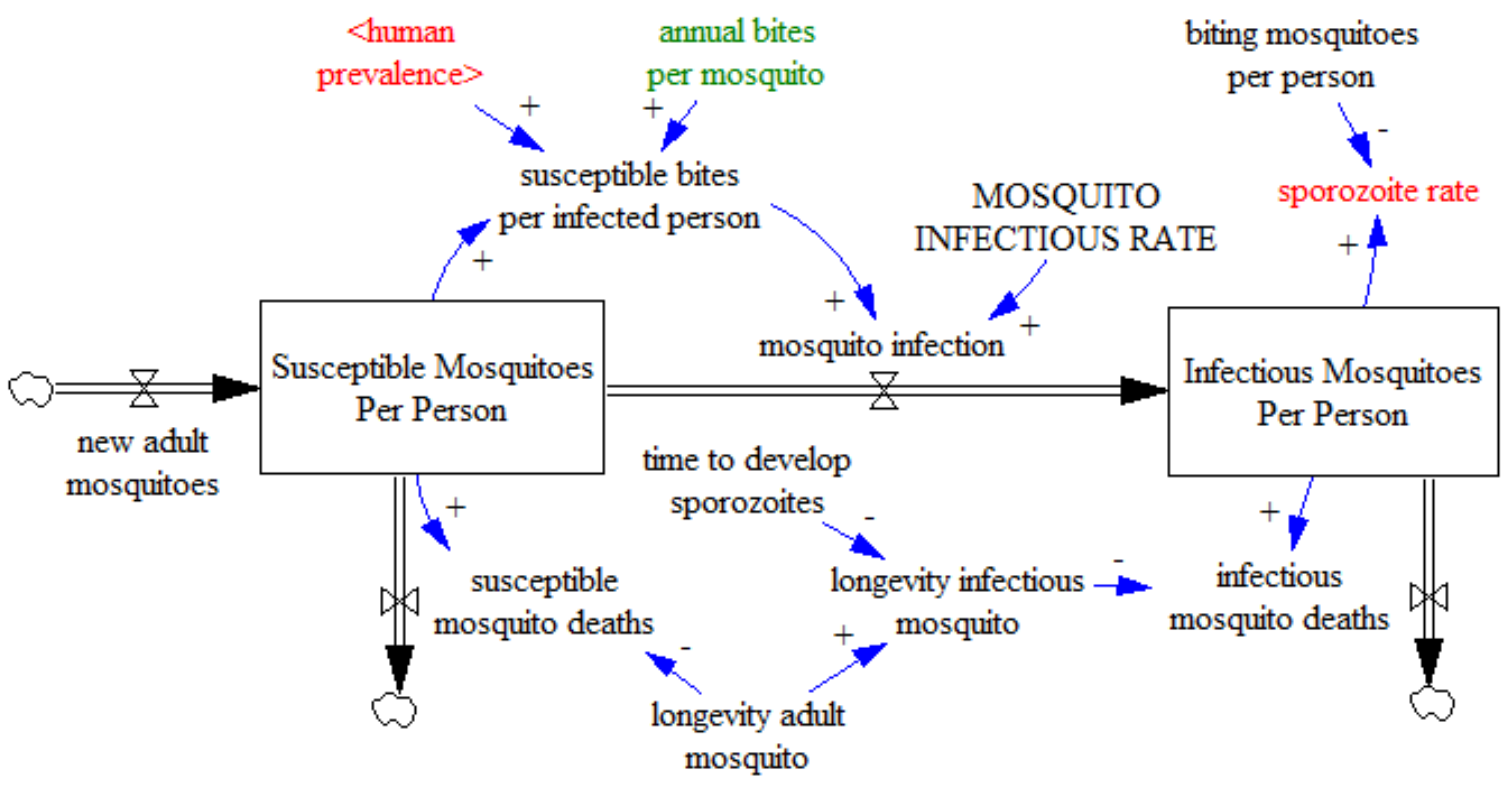

Figure 07

This sector is connected to the human sector through two indicators: From the human sector we import human prevalence and from the mosquito sector we export mosquito prevalence (sporozoite rate). These are actually the key parameters from the human and mosquito sector respectively.

Note that while in the human sector the stocks include all the persons in the area of study, in this sector the stocks only include mosquito densities in units of mosquitoes per person, and as we mentioned, we only refer to malaria-bearing anophelines i.e. female adult Anopheles mosquitoes that can potentially transmit the malaria parasite.

The dynamics of this sector basically portray the transition of adult mosquitoes from being susceptible to being infectious, and their deaths regardless of their infective state. We assume a temporally constant density of mosquitoes per person in equilibrium, which implies that the number of mosquitoes dying is approximately equal to the emerged new mosquitoes. We achieve constant mosquito density in the model by replacing the dying mosquitoes with new adult mosquitoes. An increase or reduction of the mosquito density can take place by many factors, such as variation in rains or changes in the implementation of malaria control interventions. In a more complex version of the model it is possible to implement Integrated Vector Management (IVM) interventions [32] https and see what the outcome is. For example, spraying operations can kill indoor mosquitoes reducing their average longevity, while 
bed net usage can reduce the indoor vector biting rate by reducing the bites of indoor mosquitoes to humans.

In summary, unless additional interventions are implemented or deployed, we will consider a temporally constant mosquito density. In order to keep the densities in equilibrium, the stocks will be constant over time by making the inflows equal to the outflows. Therefore, the inflow of new adult mosquitoes will be equal to the sum of mosquito deaths.

In order to initialize the value of the two stocks, we need to calculate mathematically the initial total biting mosquitoes per person $\mathrm{M}$ and then assign to each stock the corresponding part of these mosquitoes.

Once we know the value of $\mathrm{M}$, and knowing also the mosquito prevalence $\mathrm{P}_{\mathrm{M}}$ from observation, we can obtain the initial value of susceptible and infectious mosquitoes per person respectively by using the equations (08) and (09):

Susceptible Mosquitoes per Person $=M_{S}=M \cdot\left(1-P_{M}\right)$

Infectious Mosquitoes per Person $=M_{I}=M \cdot P_{M}$

With the stock values we can calculate the mosquito deaths, which are the outflows in this sector. The susceptible mosquito deaths are equal to the stock of susceptible mosquitoes $M_{S}$ divided by the longevity of adult mosquitoes $T_{M}$, and the infectious mosquito deaths are equal to the stock of infectious mosquitoes $M_{1}$ divided by the longevity of infectious mosquitoes $T_{1}$ that we calculated in (10). Substituting susceptible and infectious mosquitoes from (11), we get the next equations for mosquito deaths:

Susceptible Mosquito Deaths $=\frac{M_{S}}{T_{M}}=\frac{M \cdot\left(1-P_{M}\right)}{T_{M}}$

Infectious Mosquito Deaths $=\frac{M_{I}}{T_{I}}=\frac{M \cdot P_{M}}{T_{I}}$

The units for mosquito deaths are in mosquitoes/(person-year).

As we have indicated, in temporal steady state all the mosquito deaths are replaced or substituted by new adult mosquitoes that emerge at the same rate. In this situation the mosquito death rate would be equal to the rate of new adult mosquitoes. Given that we are talking of a fractional death rate with units in $1 /$ year, this term would be also valid for all the mosquitoes (not only mosquitoes per person), being representative of the current breeding and development capacity of the Anopheles mosquitoes in the area.

In order to calculate the total biting mosquitoes per person $\mathrm{M}$, we will operate similarly as we did in the human sector, where we also considered the system in temporal steady state.

If we focus on the stock of infectious mosquitoes represented in figure 07 , the equilibrium is reached when the mosquito infection inflow is the same as the outflow of infectious mosquito deaths, keeping constant the stock value $M_{1}$. 
The flow of mosquito infections is produced by means of susceptible mosquito bites on infected humans. In order to obtain the flow of mosquito infection, we need to calculate first the number of susceptible bites to each infected human.

Taking as a reference the stock of susceptible mosquitoes per person $\mathrm{M}_{\mathrm{s}}$, the susceptible mosquito bites per person is obtained as the product of all susceptible mosquitoes per person times the bites per mosquito $B_{R}$. Substituting $B_{R}$ from (07) and $M_{S}$ from (09) we get the next equation:

Susceptible Bites per Person $=\mathrm{M}_{\mathrm{S}} \cdot B_{R}=M \cdot\left(1-P_{M}\right) \cdot \frac{V_{R}}{M}=\left(1-P_{M}\right) \cdot V_{R}$

We observe from the result in (13) that the number of susceptible bites per person is actually the complementary of the infectious bites per person, defined in (01) as $P_{M} \cdot V_{R}$, and equal to the entomological inoculation rate. The sum of susceptible bites plus infectious bites in this model would comprise the total number of bites per person $\left(V_{R}\right)$.

From all those mosquito bites we only want the bites performed on infected humans. Therefore, we need to multiply the resulting susceptible bites by the human prevalence, which is the fraction of infected humans. At this point we assume that all humans are the same attractive to mosquitoes. If infected humans are more attractive we would need to increase the proportion of bites on these people by adding a factor. Otherwise, the resulting expression of susceptible mosquito bites per infected person $\left(S_{1}\right)$ is shown in (14).

$S_{I}=$ Susceptible Bites per Infected Person

$S_{I}=$ Susceptible Bites per Person $\cdot P_{H}=\left(1-P_{M}\right) \cdot V_{R} \cdot P_{H}$

The last step in order to calculate the flow of mosquito infections is to consider the mosquito infectious rate $I_{R}$, which is the probability of a susceptible mosquito to become infectious after biting an infected human.

So based on this probability, from all the bites made by susceptible mosquitoes on infected humans, we only consider the fraction that becomes infectious.

Therefore, the flow of mosquitoes becoming infectious is equal to the mosquito infectious rate $I_{R}$, multiplied by the susceptible bites per infected person $\mathrm{S}_{\text {I }}$. The resulting equation is represented in (15) and the units are mosquitoes/(person·year).

Mosquito Infection $=I_{R} \cdot S_{I}=I_{R} \cdot\left(1-P_{M}\right) \cdot V_{R} \cdot P_{H}$

Coming back to the consideration of having the system in equilibrium, the stock of infectious mosquitoes will keep constant when its outflow is equal to its inflow. Therefore, the infectious mosquito deaths must be the same as the mosquito infection. Using the equations (12) and (15) and substituting we get the following expression that returns the mosquitoes per person $\mathrm{M}$, also known as the ratio of mosquitoes to humans: 
Infectious Mosquito Deaths $=$ Mosquito Infection $\Rightarrow$

$$
\Rightarrow \frac{M \cdot P_{M}}{T_{I}}=I_{R} \cdot\left(1-P_{M}\right) \cdot V_{R} \cdot P_{H} \Rightarrow\left[M=\frac{T_{I} \cdot I_{R} \cdot V_{R} \cdot P_{H} \cdot\left(1-P_{M}\right)}{P_{M}}\right]
$$

We observe in (16) that the biting mosquitoes per person $\mathrm{M}$ are calculated from indicators that can be obtained empirically; some of them directly from observation $\left(V_{R}, P_{H}\right.$ and $\left.P_{M}\right)$, and the other two $\left(T_{1}\right.$ and $I_{R}$ ) indirectly, using other indicators that were also obtained by observation (the average longevity of infectious mosquito $T_{1}$ is obtained in (10), and the infectious rate is derived from the human infectivity $I_{H}$. (The mosquito sector including the infection process includes how to calculate $I_{R}$ using $I_{H}$ as a reference).

Once obtained $\mathrm{M}$, and knowing the sporozoite prevalence, we can go back to the equations in (11) and set the initial value of the stocks.

In addition, we can obtain the annual bites per mosquito $B_{R}$ by substituting (07) in (16):

$$
\left.\begin{array}{l}
M=\frac{T_{I} \cdot I_{R} \cdot V_{R} \cdot P_{H} \cdot\left(1-P_{M}\right)}{P_{M}} \\
V_{R}=B_{R} \cdot M \Rightarrow B_{R}=\frac{V_{R}}{M}
\end{array}\right\} \Rightarrow B_{R}=\frac{P_{M}}{T_{I} \cdot I_{R} \cdot P_{H} \cdot\left(1-P_{M}\right)}
$$

As we mentioned before, the intensity of malaria transmission is expressed through the EIR, which is equal to the vector biting rate times the mosquito prevalence (equation (01) where EIR $=V_{R} \cdot P_{M}$ ). In turn, from (07) $\left(V_{R}=B_{R} \cdot M\right)$, the vector biting rate $V_{R}$ depends on both, $B_{R}$ and the mosquito density $M$. Therefore the EIR can be expressed in the following way:

$$
\left.\begin{array}{l}
E I R=V_{R} \cdot P_{M} \\
V_{R}=B_{R} \cdot M
\end{array}\right\} \Rightarrow E I R=B_{R} \cdot M \cdot P_{M}
$$

With the EIR obtained in (18) and the infectious mosquitoes per person $M_{1}$ from (11), we get the following equation:

$$
\left.\begin{array}{l}
E I R=B_{R} \cdot M \cdot P_{M} \\
M_{I}=M \cdot P_{M}
\end{array}\right\} \Rightarrow E I R=B_{R} \cdot M_{I}
$$

In (19) we have deduced an alternative formula for EIR, determined as the annual bites per mosquito $B_{R}$ multiplied by the infectious mosquitoes per person $\mathrm{M}_{1}$. This multiplication logically results in the annual infectious bites received by any individual, which is precisely the definition of annual EIR.

Finally, similarly as we calculated in (04) the equation for human prevalence, from (07) and (18) we can also obtain the mosquito prevalence depending on indicators from the mosquito sector: 


$$
\begin{aligned}
& \left.\begin{array}{l}
V_{R}=B_{R} \cdot M \\
M=\frac{T_{I} \cdot I_{R} \cdot V_{R} \cdot P_{H} \cdot\left(1-P_{M}\right)}{P_{M}}
\end{array}\right\} \Rightarrow M \cdot P_{M}=T_{I} \cdot I_{R} \cdot B_{R} \cdot M \cdot P_{H} \cdot\left(1-P_{M}\right) \Rightarrow \\
& \Rightarrow P_{M}+T_{I} \cdot I_{R} \cdot B_{R} \cdot P_{H} \cdot P_{M}=T_{I} \cdot I_{R} \cdot B_{R} \cdot P_{H} \Rightarrow\left[P_{M}=\frac{T_{I} \cdot I_{R} \cdot B_{R} \cdot P_{H}}{1+T_{I} \cdot I_{R} \cdot B_{R} \cdot P_{H}}\right]
\end{aligned}
$$

The expression in (20) is similar to (04), when we calculated human prevalence related to EIR. Both equations are in the format of $y=K x /(1+K x)$.

From (20) we can plot the relationship between mosquito and human prevalence in the next graph (Figure 08), where we assume the following values: $V_{R}=365$ bites/(person·year), $M=20$ mosquitoes/person, $T_{1}=4$ days ( 0.011 years) and $I_{R}=15 \%$ ( 0.15 mosquitoes/bite).

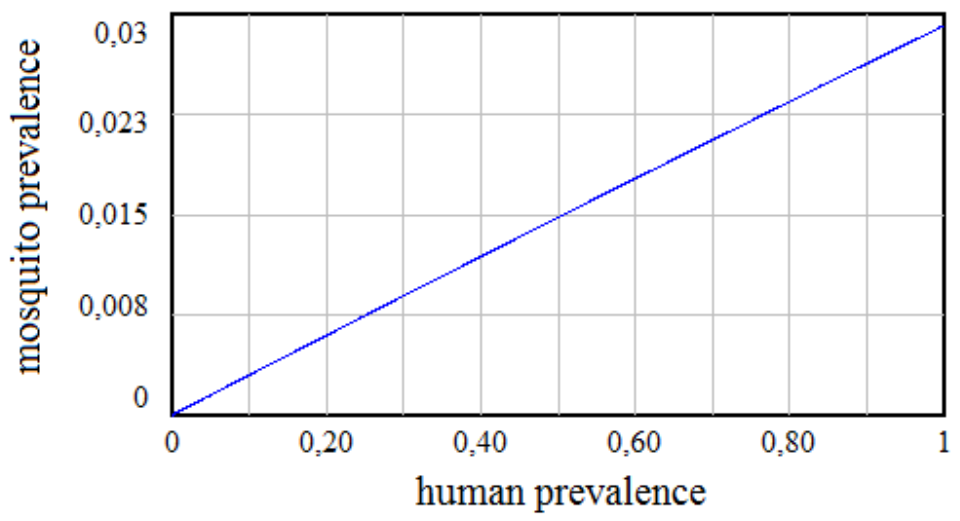

Figure 08

We observe in figure 8 that for the given values of the indicators, the maximum mosquito prevalence would be $2.9 \%$, which is reached when the area of study becomes holoendemic (essentially every individual in the population is infected or the human prevalence is equal to 1 ).

Although the expressions for human prevalence (04) and mosquito prevalence (20) have the same format, their corresponding graphs (Figure 04 and figure 08) look different. Since the human prevalence can only range from 0 to 1 , the function only works in an interval where the relationship between human and mosquito prevalence can be considered linear.

This linearity is due to the fact that even in very favorable conditions for malaria transmission, the term $\left(T_{1} \cdot I_{R} \cdot B_{R}\right)$ is very small compared to 1 : Using the values from the previous example, we obtain $B_{R}$ using the equation (07), where $B_{R}=V_{R} / M=18.25$ bites/(mosquito $\left.\cdot y e a r\right)$, therefore the term $T_{1} \cdot I_{R} \cdot B_{R}$ is equal to 0.03 , which is very small compared to 1 .

In addition, $\mathrm{P}_{\mathrm{H}}$ only ranges from 0 to 1 , therefore we can make the following approximation: 
$\left.\begin{array}{l}P_{M}=\frac{T_{I} \cdot I_{R} \cdot B_{R} \cdot P_{H}}{1+T_{I} \cdot I_{R} \cdot B_{R} \cdot P_{H}} \\ T_{I} \cdot I_{R} \cdot B_{R}<<1 \text { and } 0<P_{H}<1\end{array}\right\} \Rightarrow P_{M} \approx T_{I} \cdot I_{R} \cdot B_{R} \cdot P_{H}$

From this expression we can obtain the approximated relationship between Plasmodium falciparum prevalence in humans and mosquitoes:

$\left[\frac{P_{M}}{P_{H}} \approx T_{I} \cdot I_{R} \cdot B_{R}\right]$ where $T_{I}=\frac{T_{M}-T_{P}}{2}$

As a result, if we consider the term $T_{1} \cdot I_{R}$ as a constant that depends on the entomological and environmental conditions of the area, the ratio $\mathrm{P}_{\mathrm{M}} / \mathrm{P}_{\mathrm{H}}$ is then proportional to the average bites on humans per mosquito $B_{R}$. This also means that for a given human prevalence $P_{H}$, the mosquito prevalence $P_{M}$ is proportional to $B_{R}$.

As a summary, the presented analysis has derived the equations that would allow us to obtain the main root indicators for malaria transmission, which are $V_{R}, B_{R}, M, P_{H}$ and $P_{M}$. Based on the established relationships from the equations, we are able to eliminate three degrees of freedom; one from the human sector, one from the mosquito sector, and another one from the equation that links both sectors, relating $\mathrm{P}_{\mathrm{H}}$ and $\mathrm{P}_{\mathrm{M}}$. This means that we could obtain the value for the five indicators by knowing only two of them.

Finally, based on the equations we can observe that all the indicators are obtained regardless of human population $\mathrm{H}$. Therefore, the results are insensitive to the number of people and to different temporal steady state scenarios where human population has increased or decreased.

\subsection{Mosquito Sector including the Infection Process.}

The main purpose of including the mosquito infection process is to determine the mosquito infectious rate $\left(I_{R}\right)$, and the mathematical relationship with the human infectivity $\left(I_{H}\right)$.

The new diagram includes the transitory stock of infected mosquitoes per person and can be seen in the figure 09. Now some of the susceptible mosquitoes that bite infected humans initiate the infection process depending on the human infectivity and go to the stock of infected mosquitoes where they remain until they die or until they become infectious after the time to develop sporozoites $\left(T_{p}\right)$. After developing sporozoites, which move to the salivary glands of the female Anopheles mosquito, mosquitoes become infectious, and in the model they move to the stock of infectious mosquitoes. Infectious mosquitoes are the only ones that can pass the parasite on to a susceptible human in the course of taking a blood meal. 


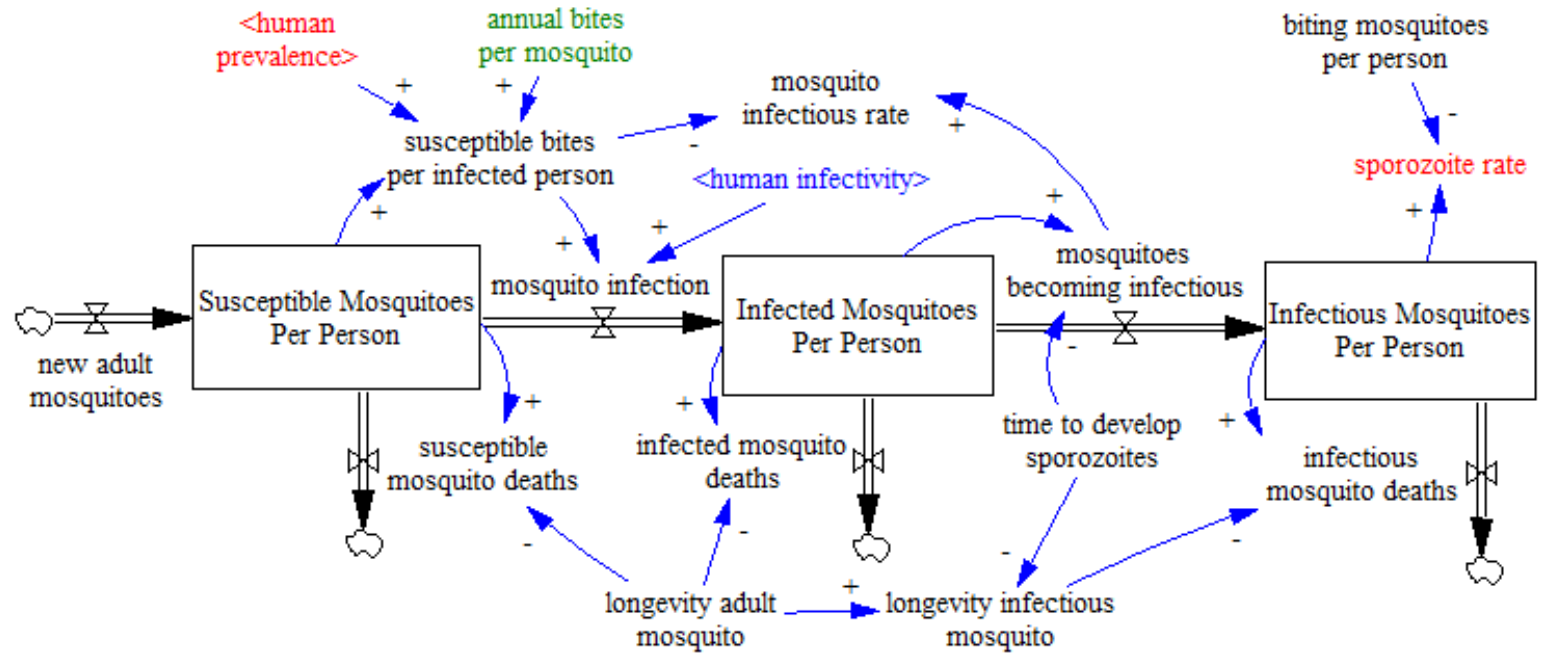

Figure 09

Like in the previous model, the value of susceptible mosquitoes per person is $\mathrm{M}_{\mathrm{S}}$ and their longevity is $T_{M}$. The new added stock contains the infected mosquitoes per person $M_{E}$, and $T_{E}$ is their corresponding longevity, which we calculated before as $T_{M} / 2$. Finally, the infectious mosquitoes per person are represented by $M_{1}$, and their longevity is expressed by $T_{1}$. Therefore, the outflow of deaths in each of the three stocks is the value of the corresponding stock divided by its average longevity. As in the previous model, we are set with a habitat of mosquitoes in temporal equilibrium which means that the inflow of new adult mosquitoes is equal to the sum of the three death outflows in order to keep constant the mosquito density.

As we explained previously, human infectivity refers to the fraction of susceptible mosquitoes that, after biting an infected person, start the infection process. On the other hand, mosquito infectious rate is defined as the fraction of susceptible mosquitoes that, after biting an infected person, start the infection process and survive long enough to develop sporozoites in the salivary glands, becoming infectious.

In the previous model, the susceptible mosquitoes picking up infection go directly to the stock of infectious mosquitoes. However, in this second version of the model, the mosquitoes go through the infection process at the stock of infected mosquitoes, during which many of them might die before becoming infectious. As a result, the fraction of mosquitoes becoming infectious is significantly smaller than the fraction of mosquitoes starting the infection process, and in consequence, the mosquito infectious rate is smaller than the human infectivity.

In (14) we calculated the annual susceptible bites per infected person $\left(S_{1}\right)$, which is equally valid for this model. However, in this model the flow of mosquito infection represented in figure 09 is equal to $S_{\text {, }}$ multiplied by the human infectivity, which is the fraction of those bites that trigger mosquito infection, or also the fraction of susceptible mosquitoes becoming infected after biting an infected person. Consequently, $S_{1}$ multiplied by the human infectivity results in the flow of mosquitoes per person that start or initiate the infection process. 
$\left.\begin{array}{l}\text { Susceptible Bites per Infected Person }=S_{I} \\ \text { Human Infectivity }=I_{H}\end{array}\right\} \Rightarrow$ Mosquito Infection $=S_{I} \cdot I_{H}$

From (23), the human infectivity can be also defined as the number of mosquitoes that pick up infection, out of the total susceptible mosquitoes biting on infected humans.

Human Infectivity $=I_{H}=\frac{\text { Mosquito Infection }}{S_{I}}$

Unlike the human infectivity, the mosquito infectious rate is the number of mosquitoes becoming infectious, out of the total susceptible mosquitoes biting on infected humans.

Mosquito Infectious Rate $=I_{R}=\frac{\text { Mosquitoes Becoming Infectious }}{S_{I}}$

Taking now the stock of infected mosquitoes as the reference, and considering the equilibrium condition, the inflow to this stock has to be equal to the sum of the outflows, where the inflow is the mosquito infection and the outflows are the infected mosquito deaths, and the mosquitoes becoming infectious.

Mosquito Infection $=$ Infected Mosquito Deaths + Mosquitoes Becoming Infectious

The infected mosquito deaths are equal to the stock $M_{E}$ divided by the longevity of infected mosquitoes $\left(T_{E}\right)$, and the outflow of mosquitoes becoming infectious is equal to the stock $M_{E}$ divided by the time to develop sporozoites $\left(T_{P}\right)$ :

Infected Mosquito Deaths $=\frac{M_{E}}{T_{E}}$

Mosquitoes Becoming Infectious $=\frac{M_{E}}{T_{P}}$

Substituting (23) and (27) in (26), we get the following expression for the human infectivity $I_{H}$ :

Mosquito Infection $=$ Infected Mosquito Deaths + Mosquitoes Becoming Infectious $\Rightarrow$

$$
\Rightarrow S_{I} \cdot I_{H}=\frac{M_{E}}{T_{E}}+\frac{M_{E}}{T_{P}}=M_{E} \cdot\left(\frac{1}{T_{E}}+\frac{1}{T_{P}}\right) \Rightarrow I_{H}=\frac{M_{E}}{S_{I}} \cdot\left(\frac{T_{E}+T_{P}}{T_{E} \cdot T_{P}}\right)
$$

Similarly we can also get the equation for the mosquito infectious rate $I_{R}$ by substituting from (27) the mosquitoes becoming infectious into (25), obtaining the following equation:

Mosquito Infectious Rate $=\mathrm{I}_{R}=\frac{\text { Mosquitoes Becoming Infectious }}{S_{I}}=\frac{M_{E} / T_{P}}{S_{I}}=\frac{M_{E}}{S_{I} \cdot T_{P}}$

If we divide (28) by (29), and substitute the longevity of infected mosquitoes ( $T_{E}=T_{M} / 2$ ), we can get the relationship between human infectivity $I_{H}$ and mosquito infectious rate $I_{R}$, depending on the longevity of adult mosquito $T_{M}$ and the time to develop sporozoites $T_{p}$ : 
$\left.\begin{array}{l}\frac{I_{H}}{I_{R}}=\frac{\frac{M_{E}}{S_{I}} \cdot\left(\frac{T_{E}+T_{P}}{T_{E} \cdot T_{P}}\right)}{\frac{M_{E}}{S_{I} \cdot T_{P}}}=\frac{T_{E}+T_{P}}{T_{E}} \\ T_{E}=\frac{T_{M}}{2}\end{array}\right\} \Rightarrow \frac{I_{H}}{I_{R}}=\frac{T_{M} / 2+T_{P}}{T_{M} / 2}=\frac{T_{M}+2 \cdot T_{P}}{T_{M}}$

We can observe that the relationship between the two indicators in (30) does not depend on any prevalence or number of bites. It only depends on mosquito longevities.

Considering that the longevity of adult mosquitoes $T_{M}$ is around three weeks and the time to develop sporozoites $T_{P}$ is not less than 10 days, the human infectivity is around double what mosquito infectious rate is. In that case, only half of the mosquitoes getting infected will become infectious, the rest will die earlier. This is valid for the given values of mosquito longevity. Should those values change due to the implementation of malaria interventions or other factors, we only need to include the new values in the equation (30) in order to obtain the relationship between human infectivity and mosquito infectious rate.

\section{Force of infection, basic reproductive number, and vectorial capacity.}

In this final chapter, we will calculate the force of infection (FOI), the basic reproductive number $\left(\mathrm{R}_{0}\right)$, and the vectorial capacity (VC), using a formulation that is related to the structure of the presented model.

-Force of infection (FOI): Also known as infection rate, the FOI is defined as the number of infections per person per unit of time. The FOI counts all incident (that is, new) human malaria infections in some time interval with or without clinical symptoms, and whether or not a person is already infected https [33].

Since we consider all infections, apart from the incidence, we also need to include the re-exposures (reinfections before recovery). The FOI is therefore the result of dividing the sum of incidence and reexposures by the total population.

The incidence and the re-exposures are both included in the following equations, where $H_{1}$ and $H_{S}$ are infected and susceptible humans respectively. We will assume similar mosquito infectivity $I_{M}$ for both groups of people:

Re - exposures $=H_{I} \cdot E I R \cdot I_{M}=H \cdot P_{H} \cdot E I R \cdot I_{M}$ Incidence $=H_{S} \cdot E I R \cdot I_{M}=H \cdot\left(1-P_{H}\right) \cdot E I R \cdot I_{M}$

$F O I=\frac{\text { Re }- \text { exposures }+ \text { Incidence }}{\text { Population }}=\frac{H \cdot P_{H} \cdot E I R \cdot I_{M}+H \cdot\left(1-P_{H}\right) \cdot E I R \cdot I_{M}}{H}=E I R \cdot I_{M}$ 
Since units for FOI are annual infections per person and EIR annual infectious bites per person, we observe that the number of infections per infectious bite $\left(F O I / E I R=I_{M}\right)$ describes the efficiency of transmission from mosquito to human.

If we define the recovery rate for humans as the inverse of the recovery time $\left(1 / T_{R}\right)$, when the FOI (or infection rate) exactly balances the rate of recovery $\left(I_{M} \cdot E I R=1 / T_{R}\right)$, half of the human population is infected:

Taking equation (04) as a reference and substituting the previous equality, we obtain the demonstration:

$\left.\begin{array}{l}P_{H}=\frac{E I R \cdot I_{M} \cdot T_{R}}{1+E I R \cdot I_{M} \cdot T_{R}} \\ I_{M} \cdot E I R=1 / T_{R} \Rightarrow E I R \cdot I_{M} \cdot T_{R}=1\end{array}\right\} \Rightarrow P_{H}=\frac{1}{2}$

Alternatively, we can also obtain the FOI as the result of dividing the incidence by the susceptible population:

$F O I=\frac{\text { Incidence }}{\text { Susceptible humans }}=\frac{H \cdot\left(1-P_{H}\right) \cdot E I R \cdot I_{M}}{H \cdot\left(1-P_{H}\right)}=E I R \cdot I_{M}$

Therefore, the force of infection is a measure of the risk of a susceptible person to become infected per unit time.

- Basic reproductive number $\left(\mathbf{R}_{\mathbf{0}}\right)$ : The basic reproduction number $\mathbf{R}_{0}$, is defined as the average number of secondary cases produced by one infected individual introduced into a completely susceptible population. In other words, $R_{0}$ is the number of infections caused by an infection in an uninfected population https [34].

Since the population will rarely be completely susceptible to an infection in the real world, one way to consider all infections as if they were rising from a totally susceptible population is to include both, infections over infected humans (re-exposures), and infections over susceptible humans (incidence).

With this premise, if we divide all the infections (i.e. re-exposures and incidence) by the stock of infected population, we will obtain the average infections per infected person and per unit of time in a totally uninfected population. This is what we call effective contact rate $\beta$ :

Re - exposures $=H_{I} \cdot E I R \cdot I_{M}=H \cdot P_{H} \cdot E I R \cdot I_{M}$

Incidence $=H_{S} \cdot E I R \cdot I_{M}=H \cdot\left(1-P_{H}\right) \cdot E I R \cdot I_{M}$

Infected $=H_{I}=H \cdot P_{H}$

$\beta=\frac{\text { Re }- \text { exposures }+ \text { Incidence }}{\text { Infected }}=\frac{H \cdot P_{H} \cdot E I R \cdot I_{M}+H \cdot\left(1-P_{H}\right) \cdot E I R \cdot I_{M}}{H \cdot P_{H}}=\frac{E I R \cdot I_{M}}{P_{H}}$

From this equation we can easily deduce that $\mathrm{FOI}=\beta \cdot \mathrm{P}_{\mathrm{H}}$. Therefore, the difference between $\beta$ and $\mathrm{FOI}$ is basically that $\beta$ refers to the infection rate per infected person, while FOI refers to the infection rate per person, regardless of the person's condition. 
Knowing that an average infected person contributes with a number of infections per unit of time, this rate $\beta$ will continue for as long as the individual is infectious. Therefore, the total number of infections derived from an infected person is the result of multiplying the infections per unit of time $\beta$, by the duration of the person's infectiousness. This duration is considered to be equal to the average recovery time $T_{R}$.

Consequently, the total number of infections per infected person $R_{0}$ is equal to the individual infections per unit of time $\beta$, multiplied by the average duration of the infectiousness $T_{R}$. We calculate $R_{0}$ as follows:

$R_{0}=T_{R} \cdot \beta=\frac{T_{R} \cdot E I R \cdot I_{M}}{P_{H}}$

It is important to note that $R_{0}$ is a dimensionless number and not a rate, which would have $1 /$ time units. $\mathrm{R}_{0}$ can be also expressed as a function of the FOI:

$R_{0}=\frac{T_{R} \cdot E I R \cdot I_{M}}{P_{H}}=\frac{T_{R} \cdot F O I}{P_{H}}$

If we only want to calculate the susceptible people who get infected per infected person, we use the effective reproductive number $\mathrm{R}$, where we only consider the incidence or new infections:

$R=\frac{T_{R} \cdot \text { Incidence }}{\text { Infected }}=\frac{T_{R} \cdot\left(H \cdot\left(1-P_{H}\right) \cdot E I R \cdot I_{M}\right)}{H \cdot P_{H}}=\left(1-P_{H}\right) \cdot \frac{T_{R} \cdot E I R \cdot I_{M}}{P_{H}}=\left(1-P_{H}\right) \cdot R_{0}$

We observe that $R$ is equal to $R_{0}$ when $P_{H}=0$, i.e. when all the population is susceptible.

In general, for an epidemic to occur in a susceptible population $R_{0}$ must be $>1$, so the number of infected people is increasing. However, $\mathrm{R}$ is more appropriate than $\mathrm{R}_{0}$ to verify whether a disease is spreading or not, because it takes into account that not everybody is susceptible.

For example, if $R_{0}$ is equal to 2 but the prevalence is $60 \%$, the number of new infections per infected person is $\mathrm{R}=(1-0.6) \cdot 2=0.8$, which means that for every infected person who gets recovered, there will be 0.8 new infections from the stock of susceptible. Given that in this situation the number of recoveries is bigger than the incidence, the prevalence will decrease over time.

Therefore, only when the effective reproductive number $\mathrm{R}>1$ (each infected person contributes with more than one new infection from the susceptible population), the number of infected people or prevalence is increasing.

If $R=1$, the prevalence is constant, and if $R<1$, the prevalence will decrease.

In the following equation we demonstrate that $\mathrm{R}>1$ when incidence $>$ recoveries: 
Incidence $=H \cdot\left(1-P_{H}\right) \cdot E I R \cdot I_{M} \quad$ Recovering $=\frac{H \cdot P_{H}}{T_{R}}$

if Incidence $>$ Recovering $\Rightarrow H \cdot\left(1-P_{H}\right) \cdot E I R \cdot I_{M}>\frac{H \cdot P_{H}}{T_{R}} \Rightarrow\left(1-P_{H}\right) \cdot \frac{T_{R} \cdot E I R \cdot I_{M}}{P_{H}}=R>1$

- Vectorial capacity (VC): The vectorial capacity VC is intimately related to the effective contact rate $\beta$. It also represents the average number of humans infected per infected human and per unit of time in a completely susceptible human population, but in this case assuming perfect transmission efficiency, i.e. $I_{M}=I_{R}=1$.

The reason to assume perfect transmission efficiency is that the VC is a concept that describes the maximum potential of a vector to transmit a pathogen https [35]. Given that in the real world there is not perfect transmission efficiency, we can define $\mathrm{VC}$ as the total number of potentially infectious bites that would eventually arise from all the mosquitoes biting a single perfectly infectious (i.e. all mosquito bites result in infection) human on a single day https [36] (Note that all our rates are in annual terms and this definition uses day as time units. In any case, we can convert annual values into daily dividing by 365$)$.

Since $I_{M}$ and $I_{R}$ are normally $<1$, the vectorial capacity $V C$ is bigger than $\beta$. Taking equation (34) as a reference, we obtain VC:

$V C=\frac{\beta}{I_{M} \cdot I_{R}}=\frac{E I R \cdot I_{M}}{P_{H} \cdot I_{M} \cdot I_{R}}$

Once we put in transmissibility $\left(I_{M}\right.$ and $\left.I_{R}\right)$ and the duration of infectiousness $T_{R}$, we have a measure directly analogous to $R_{0}$. Therefore, the basic reproduction number $R_{0}$ can be also expressed as a function of VC:

$R_{0}=T_{R} \cdot \beta=T_{R} \cdot V C \cdot I_{M} \cdot I_{R}$

Some authors use an alternative definition for vectorial capacity; the number of infectious bites that would eventually arise from all the mosquitoes that bite a fully infectious human per unit of time [4]. This would be equal to $V C$, multiplied by the transmission efficiency $I_{R}$.

\section{Discussion.}

The model presented in this paper aims for a better understanding of the mechanisms involved in malaria transmission. We have started from the simplest model version, and despite the added complexity in the different chapters, these versions are still generic and have some limitations.

During the development of the present model we have already mentioned different limitations. In this regard, the model contains the essential mechanisms of malaria transmission, but is missing other sectors like a mosquito breeding sector, or effects, like the effects of the environment in mosquito development, parasite transmission, etc., which are included in more complex versions of the model. Another limitation is that we have considered the system homogeneous: 
For most of the indicators, the presented models use average values and do not make differentiations among humans, mosquitoes, or even $\mathrm{P}$. falciparum parasites. In this primary version, we have simplified the model by considering homogeneous mosquito populations or parasite infectivity (ability of sporozoites to invade the liver of the host). Also in line with the mosquitoes, we have not included the possibility of different Anopheles species transmitting malaria with different biting behaviors, and even mosquitoes from the same species do not have the same biting patterns; for example, infectious stage mosquitoes normally increase their motivation to bite, increasing also their share of the total bites and changing their behavioral response against insecticides https [37].

Regarding humans, we have considered all the persons in an area to be similarly attractive to mosquito bites, although for instance, pregnant women can attract more mosquito bites https [38]. Moreover, malaria infection can render humans more attractive to Anopheles mosquitoes than uninfected people https [39], making the infected people to have also a bigger share of the total bites. In fact, evidence suggests that in some locations a core group of the human population receive a substantial proportion of mosquito bites because of proximity to larval habitat or differential attractiveness to mosquitoes (for example, up to $80 \%$ of the bites might be taken on just $20 \%$ of the population), increasing the level of heterogeneity https [40].

The homogenization of these and other model variables can lead to a certain level of inaccuracy in the results, but yet the models can be close enough to reality and help to understand the malaria transmission in the area of analysis. For more accurate results, the models are flexible to add more complexity, which is strongly subordinate to the ability to find the necessary data that is required. Therefore, most of the limitations can be overcome by adding the necessary level of detail, although the difficulty leans mostly upon finding reliable data. In fact, the quality of the statistical information available regarding malaria in Sub-Saharan regions is usually very poor https [41] https [42].

But even in the absence of some data, sometimes it is possible to extrapolate values from other similar locations, use the literature, or calculate them directly in the model. Nevertheless, the purpose of the model is what in the end will determine the needed level of detail or precision.

In this regard, one of the purposes of malaria models is to design adequate malaria control programs in the areas of study, and one of the most successful ways to plan malaria control policies is through the use of integrated vector control management (IVM) [43] https. Since IVM works reducing malaria transmission using different interventions, the models require differentiation between malaria transmission outdoors and indoors. To that extent the model can be enlarged in order to capture the different dynamics between indoor and outdoor mosquitoes.

We do not elaborate mathematically on the impact of IVM interventions in this paper but if we assume the inclusion of indoor and outdoor sectors in the model, we can at least indicate briefly the dynamics of the different interventions in our model:

As mentioned before, bed net usage can reduce the indoor vector biting rate. And since the nets restrict the access to indoor blood meals, the mosquitoes will try to find another way to bite hosts, which in return can eventually affect the peak biting hours [44] https, the outdoor biting rate, and also the 
human blood index [45] https. A decrease in the human blood index would decrease proportionally the annual bites on humans per mosquito $B_{R}$.

Nowadays most nets are treated (Long Lasting Insecticide-treated Nets, or simply LLINs), and they can therefore contribute to kill some of the mosquitoes. The same applies to IRS (Indoor Residual Spraying), which reduces both, the longevity of indoor mosquitoes and the willingness of mosquitoes in general to get indoors (excito-repellency that reduces the number of indoor mosquitoes per person). We have to consider however, that the efficacy of these methods is highly dependent on the mosquito resistance to the insecticides.

Regarding outdoor interventions, environmental management operations, including source reduction or larviciding [46] https, can reduce the inflow of new adult mosquitoes into the environment, reducing also mosquito density and consequently the number of biting mosquitoes per person. If we assume a constant number of annual bites per mosquito $B_{R}$, a decrease in the number of biting mosquitoes per person $M$ would reduce proportionally the vector biting rate $V_{R}$ (based on equation (07), where $\left.V_{R}=B_{R} \cdot M\right)$.

The reduction in mosquito density and bites per person after implementing the different interventions can be obtained empirically. These effects can be included in the model, allowing a holistic assessment of the combined impact from different IVM interventions in reducing malaria transmission.

Should the interventions reduce significantly the incidence, the prevalence and the level of re-exposures would be also reduced. Consequently, the fraction of asymptomatic population would decrease over time, which in turn would decrease also the recovery time (assuming proper case management and prompt treatment).

In our case, the recovery time comprises the period for a complete elimination of malaria parasites from the body (we do not use the term "radical cure" as it is only used for P. vivax and P. ovale infections to reflect the use of anti-hypnozoite medicines). Therefore, a decrease in the recovery time implies a shorter infectious period, which reduces the availability of parasites in humans and results in a lower human prevalence.

As a result, a decrease in human prevalence would lead to a reduction in the mosquito infection rate and the consequent reduction in mosquito prevalence, which in turn would lead to a smaller EIR and further reduction in human prevalence. This major feedback loop is represented with red arrows in the figure 10, where we have put together the basic human sector (figure 03) and mosquito sector (figure 07). 


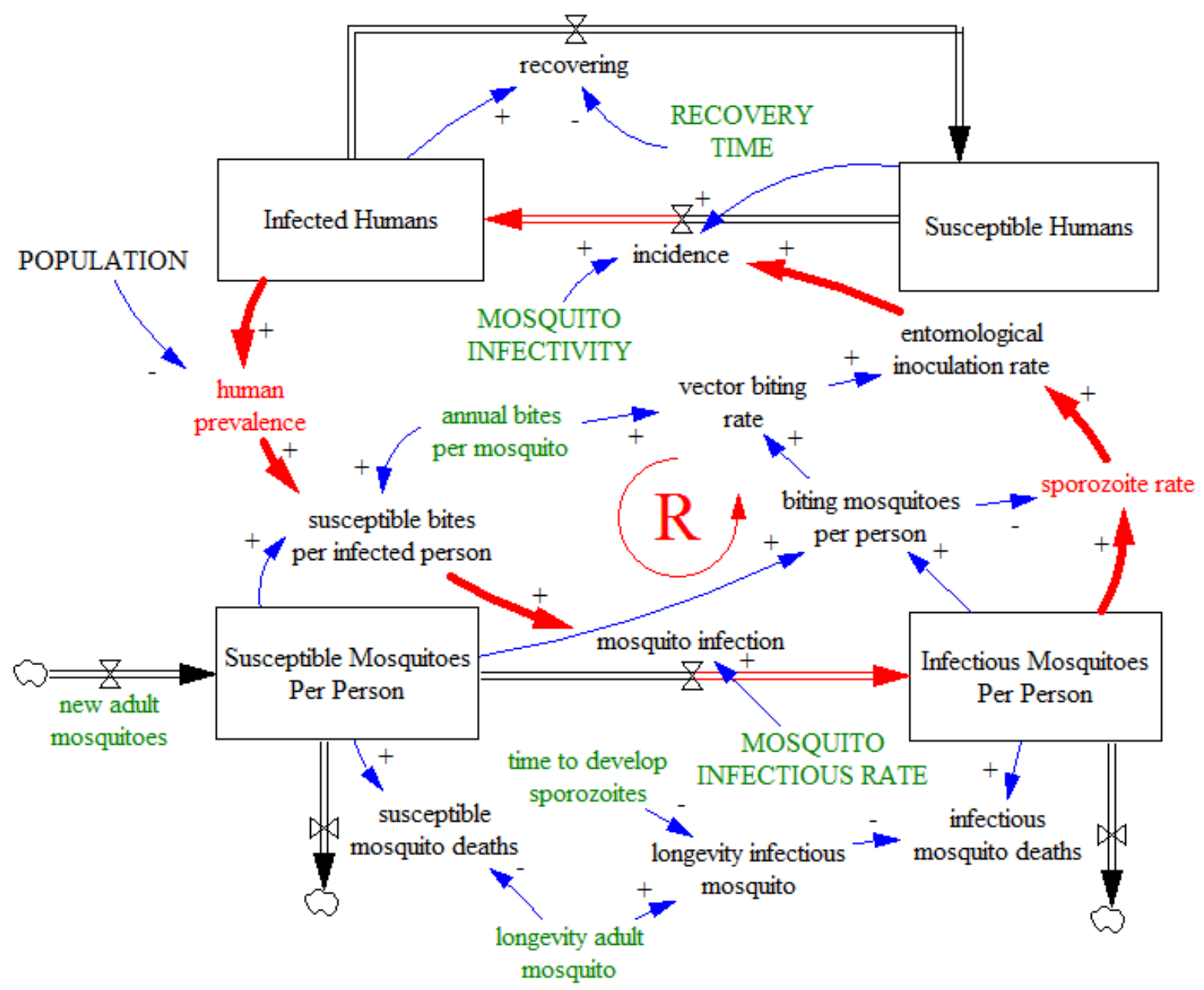

Figure 10

If for example we assume a significant increment in the number of new adult mosquitoes, this disturbance would break the steady state of the system, and the major feedback loop could trigger rapid increases in human prevalence leading to an epidemic situation. On the contrary, if the transmission is compromised by a very low density of humans or mosquitoes carrying the parasite, the loop can develop into malaria elimination. In fact, should the prevalence be reduced under some thresholds, malaria transmission would become unsustainable and be eliminated. In that situation, the obtained equilibrium value for any prevalence in the model would reach marginal values that are in practice equal to zero.

Therefore, this major feedback loop aims to bring the system into extreme situations $\left(\mathrm{P}_{H}=1\right.$ or $\left.\mathrm{P}_{\mathrm{H}}=0\right)$. Given its nature, we define the major feedback loop as reinforcing, in agreement with the System Dynamics theory. The loop is actually symbolized in the figure 10 by a big letter $\mathrm{R}$ surrounded by the logo of a circular arrow which indicates the direction of the causality.

The indicators colored in green are those that can vary through external factors, playing a fundamental role in defining the behavior of this loop. However, for the purpose of simplicity, we do not represent the effects of the different malaria control interventions that we have mentioned before. 
Since malaria transmission is also subject to balancing feedbacks, the system needs to include other counteracting loops that prevent the prevalence from reaching extreme values and allow temporal steady states when the forces of the system are in equilibrium. For example, the recovery of humans decreasing the stock of infected humans, or the mosquito deaths decreasing the stock of mosquitoes are part of other balancing loops that impede our major feedback loop from increasing malaria prevalence uncontrolled. Other examples of balancing loops can be found by analyzing the transmission efficiency or infectivity:

For example, several studies that have estimated the mosquito infectivity $I_{M}$ have shown that mosquitoto-human transmission is extremely inefficient in high-intensity settings (high EIR). On the contrary, as malaria prevalence falls, transmission dynamics and immunoepidemiology change and infected mosquitoes are more likely to transmit the infection [33].

Something very similar happens with the human-to-mosquito transmission efficiency $I_{H}$. In highly endemic areas, a small fraction of mosquitoes biting an infected host develop sporozoites, but as malaria prevalence becomes smaller, the human infectivity increases https [47].

Regardless of the reasons that relate human prevalence with infectivity, there is a causality indicating that a higher prevalence will lead to lower mosquito infectivity. In this case, incidence would become smaller, which in return would reduce prevalence, generating a balancing loop as indicated in figure 11.

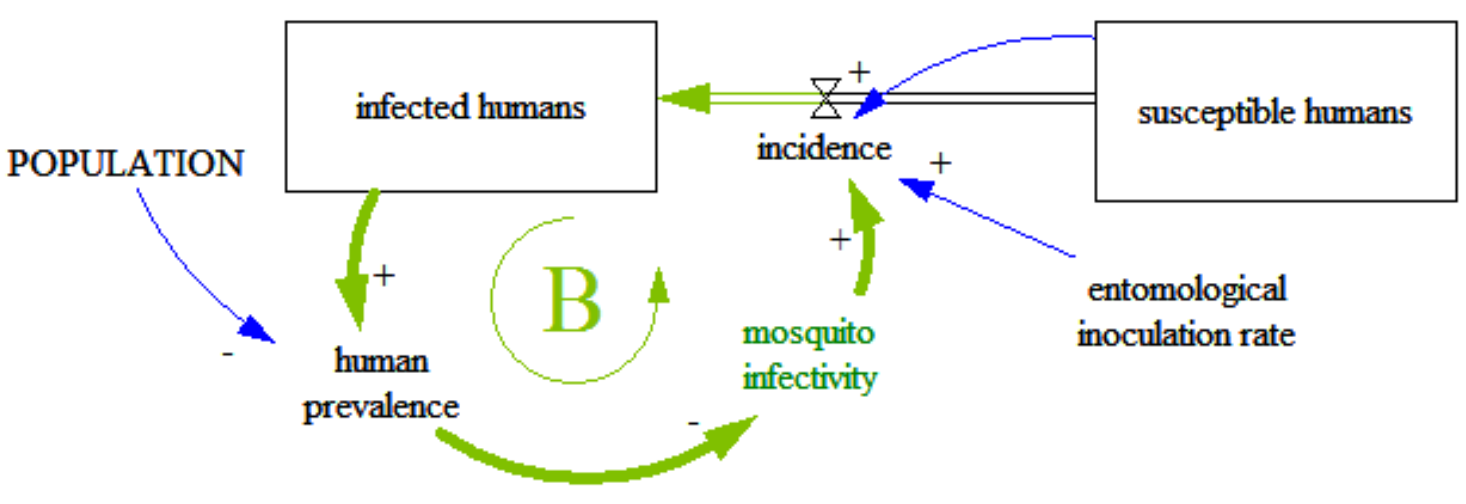

Figure 11

Depending on how much human prevalence affects mosquito infectivity, we can deduce the extent at which this change in transmission efficiency complicates the relationship between human prevalence and the EIR represented in figure 04.

A similar balancing loop is present when dealing with the human infectivity: Higher human prevalence leads to less efficient human-to-mosquito transmission, which in return decreases sporozoite rate, decreasing also incidence and human prevalence. These loops are not represented in the figure 10 but they might need to be included in the models when the prevalence in the area of analysis experiences significant variations. 
Based on the discussion so far, and in addition to all the static analysis performed under the assumption of temporal steady state, we can conclude that the presented model portrays feedback loops that can develop changes in the behavior of the system, generating different dynamics of malaria transmission. Given that we are dealing with an infectious disease, our dynamic analysis would focus on changes in the incidence or prevalence of the population over time [4].

A last consideration in interpreting our results is about the annual bites on humans per mosquito $B_{R}$ and its significance regarding malaria transmission. As we deduced from (07), any reduction of $B_{R}$ or $M$ would reduce $V_{R}$, reducing also the EIR. However, similar fractional reduction in $B_{R}$ and in $M$ does not have the same relevance:

If we substitute the vector biting rate (07) and the mosquito prevalence (20) in the equation (01) that defines the EIR, we get the following equation:

$\left.\begin{array}{l}V_{R}=B_{R} \cdot M \\ P_{M}=\frac{T_{I} \cdot I_{R} \cdot B_{R} \cdot P_{H}}{1+T_{I} \cdot I_{R} \cdot B_{R} \cdot P_{H}} \\ E I R=V_{R} \cdot P_{M}\end{array}\right\} \Rightarrow E I R=\frac{B_{R}^{2} \cdot M \cdot T_{I} \cdot I_{R} \cdot P_{H}}{1+T_{I} \cdot I_{R} \cdot B_{R} \cdot P_{H}}$

From this equation we already considered in (21) that the term $\left(1+T_{I} \cdot I_{R} \cdot B_{R} \cdot P_{H}\right)$ is approximately equal to 1 , making possible to simplify (41) by removing the denominator:

$$
E I R \approx B_{R}^{2} \cdot M \cdot T_{I} \cdot I_{R} \cdot P_{H}
$$

As we indicated, the intensity of malaria transmission is expressed through the EIR, which represents the number of infective bites received by an individual during the year. This number can be obtained in (42) by multiplying different factors, and we observe that $B_{R}$ is raised to the power of 2, which means that the EIR is significantly more sensitive to $B_{R}$ than to any other factor. In fact, variations in $M$ might not have the expected effect in the EIR since, as we suggested previously, increasing $M$ might decrease $B_{R}$ (or reducing $M$ might increase $B_{R}$ ), which would partially cancel out the effect of $M$ on the EIR.

To put an example, if we had a constant number of 10 mosquitos per person, and each of them bite a human once per week, the malaria transmission would be less intense than having 5 mosquitoes per person, each of them biting twice per week.

It is true that in both cases the total number of mosquito bites is the same, but it is also logical to think that mosquitos with higher biting activity are more likely to contract malaria and therefore transmit it to someone else, which means that for malaria transmission, $B_{R}$ is more critical than the number of mosquitoes per person $M$.

As a result, reducing $B_{R}$ plays an even more essential role in malaria reduction than reducing the mosquito density. If for example, we reduce the bites per mosquito $B_{R}$ by half, the resulting EIR would be four times smaller.

In this sense, another important factor is the human blood index: Mosquitoes having most of their blood meals from humans contribute to a higher $B_{R}$ that could trigger epidemics even when mosquito density 
$M$ has been reduced significantly. Therefore, diverting the bites on humans to other animal species can also play an important role in reducing malaria transmission.

In order to have an idea of the variations in the average bites per mosquito $B_{R}$, we can use the expression (56), where $B_{R}$ is approximately proportional to the ratio $P_{M} / P_{H}$.

\section{Conclusion.}

We have fitted a simple stock and flow model which allows us to understand the most important mechanisms of malaria transmission in P. falciparum carriers. Although the starting model is very simple, the results obtained after increasing the model complexity are very similar.

The mathematical relationships established in the model can help us to obtain or validate the value of the main malaria transmission indicators by eliminating three degrees of freedom. In this sense, one of the most relevant results from this study is the calculated ratio or relationship between mosquito and human prevalence $\left(\mathrm{P}_{\mathrm{M}} / \mathrm{P}_{\mathrm{H}}\right)$, which is approximately equal to a constant that depends on the entomological and environmental conditions of the area, multiplied by the average bites on humans per mosquito $B_{R}$.

Regarding $B_{R}$, it can be also calculated using other indicators obtained empirically and is particularly relevant for malaria transmission as it would enable high levels of human prevalence even in environments with low mosquito densities.

Although the relationships in the model are obtained from the perspective of a static analysis, the inclusion of data would allow the model to simulate over time and to manifest the behavior of the variables driven by the different feedback loops of the system, thus permitting also dynamic analyses.

So far we have developed introductory models that can help us to understand better the problem of malaria transmission. However, we have not used them yet to solve this problem. One of the next steps in this direction would be to introduce different malaria control interventions into the model, making possible to simulate them and observe their outcome over time, which in return would provide a platform from where it is possible to analyze dynamically their impact on malaria transmission. Once validated for specific locations, the models can be used as decision-support tools to improve the design of strategies against malaria in the different areas.

\section{Declarations.}

\section{Ethics approval and consent to participate.}

Not applicable.

\section{Consent for publication.}

Not applicable.

\section{Availability of data and materials.}

Not applicable. 


\section{Competing interests.}

The author declares that he has no competing interests.

\section{Funding.}

Not applicable.

\section{Authors' contributions.}

Not applicable.

\section{Acknowledgements.}

Not applicable.

\section{Reference List.}

1. Autino, B., et al., Epidemiology of malaria in endemic areas. Mediterranean journal of hematology and infectious diseases, 2012. 4(1): p. e2012060-e2012060.

2. Rosenthal, P.J., How do we best diagnose malaria in Africa? Am J Trop Med Hyg, 2012. 86(2): $p$. 192-3.

3. White, N.J., Determinants of relapse periodicity in Plasmodium vivax malaria. Malar J, 2011. 10: p. 297.

4. Smith, D.L. and F.E. McKenzie, Statics and dynamics of malaria infection in Anopheles mosquitoes. Malar J, 2004. 3: p. 13.

5. Homer, J.B. and G.B. Hirsch, System dynamics modeling for public health: background and opportunities. Am J Public Health, 2006. 96(3): p. 452-8.

6. Koella, J.C., On the use of mathematical models of malaria transmission. Acta Tropica, 1991. 49(1): p. 1-25.

7. WHO, WHO malaria terminology. 2018.

8. Kilama, M., et al., Estimating the annual entomological inoculation rate for Plasmodium falciparum transmitted by Anopheles gambiae s.l. using three sampling methods in three sites in Uganda. Malar J, 2014. 13: p. 111.

9. Kabbale, F., et al., Biting times of Plasmodium falciparum infected mosquitoes and transmission intensities following five years of insecticide-Treated bed nets use in Kamuli District, Uganda: Implications for malaria control. International Journal of Mosquito Research, 2016. Vol-3, Issue4.

10. Beier, J., G. Killeen, and J. I Githure, Short report: Entomologic inoculation rates and Plasmodium falciparum malaria prevalence in Africa. Vol. 61. 1999. 109-13.

11. Churcher, T.S., et al., Probability of Transmission of Malaria from Mosquito to Human Is Regulated by Mosquito Parasite Density in Naive and Vaccinated Hosts. PLoS Pathog, 2017. 13(1): p. e1006108.

12. Lindblade, K.A., et al., The silent threat: asymptomatic parasitemia and malaria transmission. Expert Rev Anti Infect Ther, 2013. 11(6): p. 623-39.

13. Felger, l., et al., The dynamics of natural Plasmodium falciparum infections. PLoS One, 2012. 7(9): p. e45542.

14. Birley, M.H. and J.D. Charlewood, Sporozoite rate and malaria prevalence. Parasitol Today, 1987. 3(8): p. 231-2. 
15. Ndoen, E., et al., Mosquito Longevity, Vector Capacity, and Malaria Incidence in West Timor and Central Java, Indonesia. ISRN Public Health, 2012. 2012: p. 1-5.

16. Charlwood, J.D., et al., Feeding frequency and survival of Anopheles gambiae in a rice-growing area in Ghana. Med Vet Entomol, 2012. 26(3): p. 263-70.

17. Ndiath, M.O., et al., Methods to collect Anopheles mosquitoes and evaluate malaria transmission: a comparative study in two villages in Senegal. Malar J, 2011. 10: p. 270.

18. Beier, J.C., Malaria parasite development in mosquitoes. Annu Rev Entomol, 1998. 43: p. 519-43.

19. Klowden, M.J., Blood, Sex, and the Mosquito. BioScience, 1995. 45(5): p. 326-331.

20. Graves, P.M., et al., Measurement of malarial infectivity of human populations to mosquitoes in the Madang area, Papua New Guinea. Parasitology, 2009. 96(02).

21. Gardiner, D.L. and K.R. Trenholme, Plasmodium falciparum gametocytes: playing hide and seek. Annals of translational medicine, 2015. 3(4): p. 45-45.

22. Githeko, A.K., et al., The reservoir of Plasmodium falciparum malaria in a holoendemic area of western Kenya. Trans R Soc Trop Med Hyg, 1992. 86(4): p. 355-8.

23. Ashley, E.A. and N.J. White, The duration of Plasmodium falciparum infections. Malar J, 2014. 13: p. 500.

24. Azikiwe, C.C.A., et al., A comparative laboratory diagnosis of malaria: microscopy versus rapid diagnostic test kits. Asian Pacific Journal of Tropical Biomedicine, 2012. 2(4): p. 307-310.

25. Dalrymple, U., et al., How long do rapid diagnostic tests remain positive after anti-malarial treatment? Malar J, 2018. 17(1): p. 228.

26. Nosten, F. and N. J White, Artemisinin-Based Combination Treatment of falciparum Malaria. Vol. 77. 2008. 181-92.

27. Wilairatana, P., S. Krudsood, and N. Tangpukdee, Appropriate time for primaquine treatment to reduce Plasmodium falciparum transmission in hypoendemic areas. Korean J Parasitol, 2010. 48(2): p. 179-82.

28. Mueller, I., et al., The sensitivity of the OptiMAL rapid diagnostic test to the presence of Plasmodium falciparum gametocytes compromises its ability to monitor treatment outcomes in an area of Papua New Guinea in which malaria is endemic. J Clin Microbiol, 2007. 45(2): p. 62730.

29. Bousema, T., et al., Revisiting the circulation time of Plasmodium falciparum gametocytes: molecular detection methods to estimate the duration of gametocyte carriage and the effect of gametocytocidal drugs. Malar J, 2010. 9: p. 136.

30. Arévalo-Herrera, M., et al., Malaria transmission blocking immunity and sexual stage vaccines for interrupting malaria transmission in Latin America. Memórias do Instituto Oswaldo Cruz, 2011. 106(suppl 1): p. 202-211.

31. Doolan, D.L., C. Dobano, and J.K. Baird, Acquired immunity to malaria. Clin Microbiol Rev, 2009. 22(1): p. 13-36, Table of Contents.

32. Beier, J.C., et al., Integrated vector management for malaria control. Malar J, 2008. 7 Suppl 1: p. S4.

33. Smith, D.L., et al., A quantitative analysis of transmission efficiency versus intensity for malaria. Nat Commun, 2010. 1: p. 108.

34. van den Driessche, P., Reproduction numbers of infectious disease models. Infect Dis Model, 2017. 2(3): p. 288-303.

35. Catano-Lopez, A., et al., An alternative model to explain the vectorial capacity using as example Aedes aegypti case in dengue transmission. Heliyon, 2019. 5(10): p. e02577.

36. Brady, O.J., et al., Vectorial capacity and vector control: reconsidering sensitivity to parameters for malaria elimination. Trans R Soc Trop Med Hyg, 2016. 110(2): p. 107-17.

37. Thievent, K., et al., Malaria infection in mosquitoes decreases the personal protection offered by permethrin-treated bednets. Parasit Vectors, 2018. 11(1): p. 284. 
38. Himeidan, Y.E., M.I. Elbashir, and I. Adam, Attractiveness of pregnant women to the malaria vector, Anopheles arabiensis, in Sudan. Ann Trop Med Parasitol, 2004. 98(6): p. 631-3.

39. Emami, S.N., et al., A key malaria metabolite modulates vector blood seeking, feeding, and susceptibility to infection. Science, 2017. 355(6329): p. 1076-1080.

40. Smith, D.L., et al., The entomological inoculation rate and Plasmodium falciparum infection in African children. Nature, 2005. 438(7067): p. 492-5.

41. Ohiri, K., et al., An Assessment of Data Availability, Quality, and Use in Malaria Program Decision Making in Nigeria. Health Systems \& Reform, 2016. 2(4): p. 319-330.

42. Chilundo, B., J. Sundby, and M. Aanestad, Analysing the quality of routine malaria data in Mozambique. Malar J, 2004. 3: p. 3.

43. Tizifa, T.A., et al., Prevention Efforts for Malaria. Curr Trop Med Rep, 2018. 5(1): p. 41-50.

44. Yohannes, M. and E. Boelee, Early biting rhythm in the Afro-tropical vector of malaria, Anopheles arabiensis, and challenges for its control in Ethiopia. Med Vet Entomol, 2012. 26(1): p. 103-5.

45. Killeen, G.F., Characterizing, controlling and eliminating residual malaria transmission. Malar J, 2014. 13: p. 330.

46. WHO. Malaria control: the power of integrated action. Available from: https://www.who.int/heli/risks/vectors/malariacontrol/en/index2.html.

47. Churcher, T.S., J.F. Trape, and A. Cohuet, Human-to-mosquito transmission efficiency increases as malaria is controlled. Nat Commun, 2015. 6: p. 6054. 
Figures

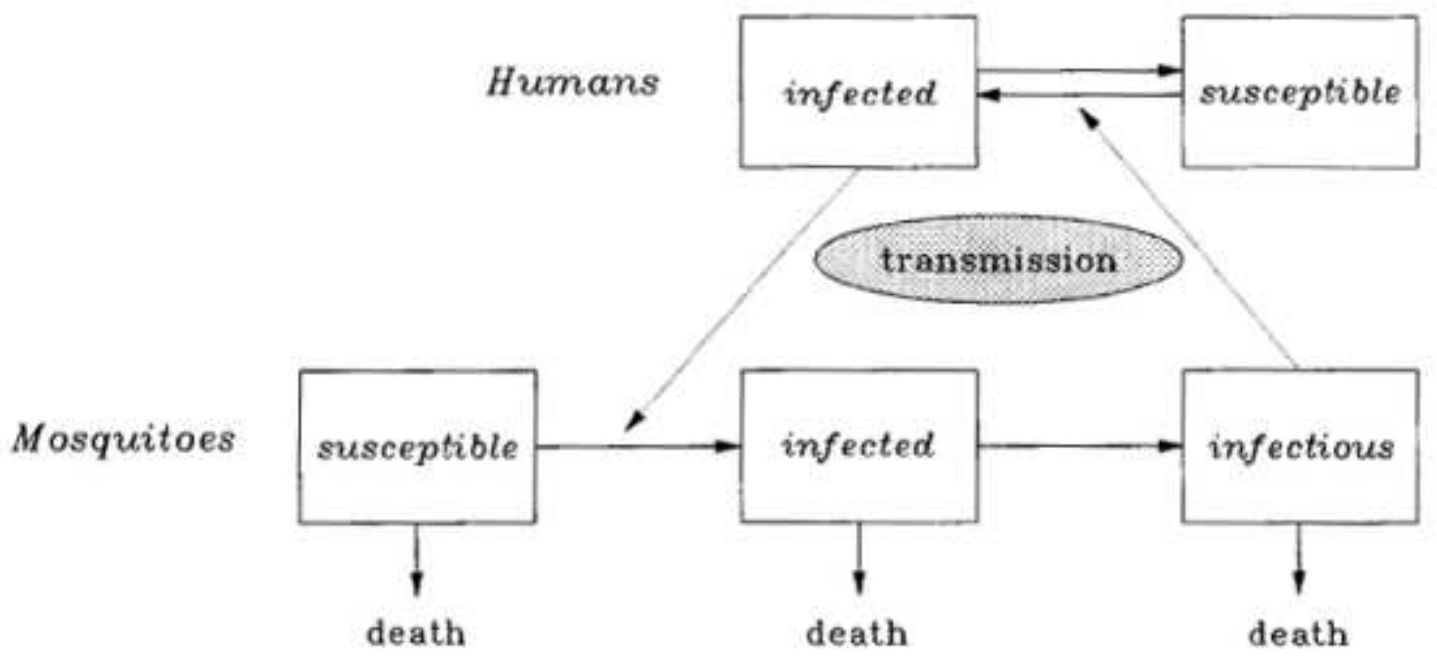

\section{Figure 1}

The structure is inspired by the Ross-Macdonald model of malaria transmission

$$
\begin{aligned}
& H=\text { Human Population } \\
& H_{S}=\text { Susceptible Humans } \\
& H_{I}=\text { Infected Humans } \\
& P_{H}=\text { Human Prevalence } \\
& P_{M}=\text { Mosquito Prevalence or Sporozoite Rate } \\
& V_{R}=\text { Vector Biting Rate } \\
& E I R=\text { Entomological Inoculation Rate } \\
& I_{M}=\text { Mosquito Infectivity } \\
& T_{R}=\text { Average Recovery Time }
\end{aligned}
$$

\section{Figure 2}

contains a list of indicators with the abbreviations that we will use for the equations 


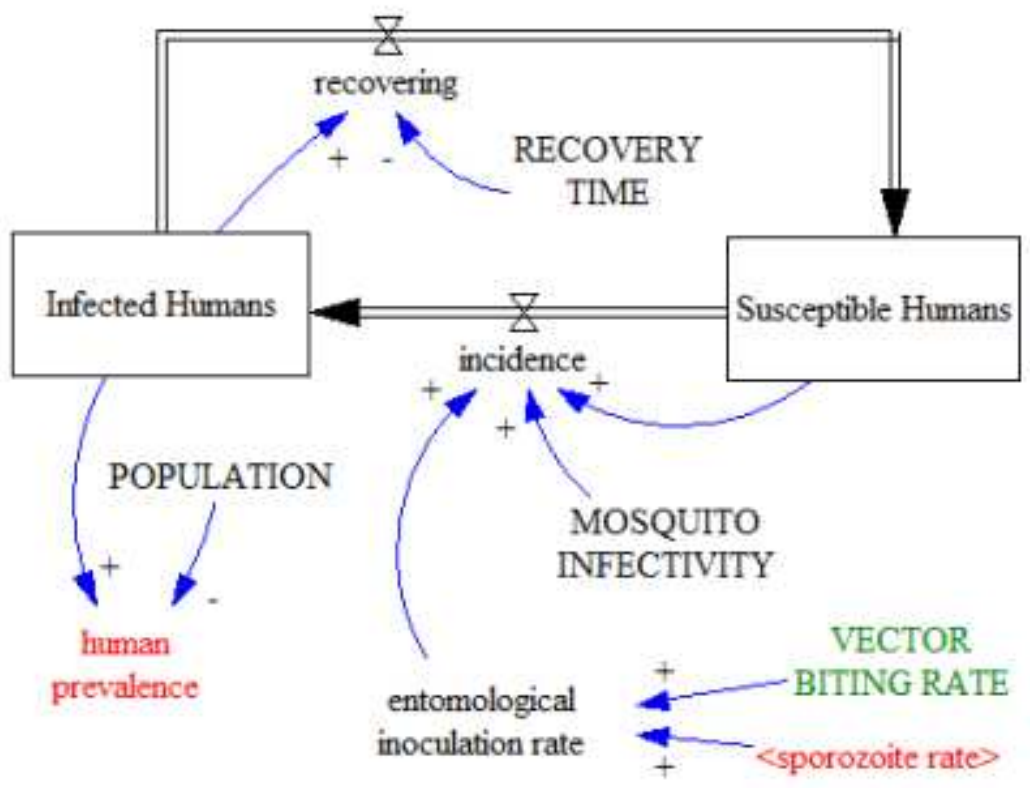

Figure 3

Once defined the first list of indicators, we can see them linked in the model diagram

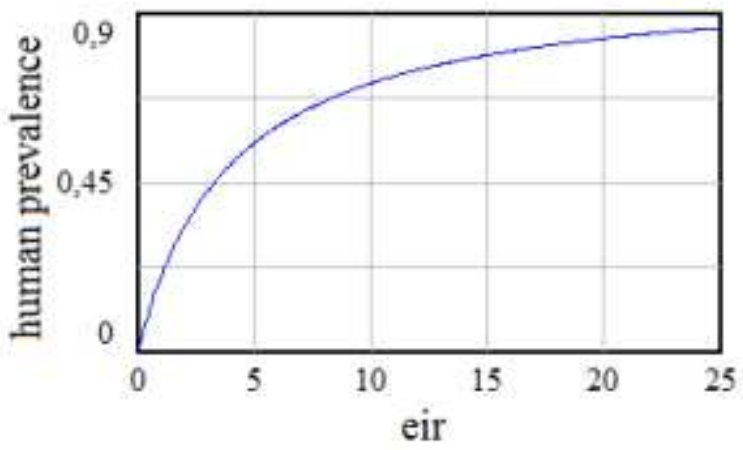

\section{Figure 4}

This relationship between EIR and the proportion of humans with parasitemia can be seen in the next graph 
$B_{R}=$ Annual Bites per Mosquito

$M=$ Biting Mosquitoes per Person

$M_{t}=$ Infectious Mosquitoes per Person

$M_{S}=$ Susceptible Mosquitoes per Person

$T_{p}=$ Time to Develop Sporozoites

$T_{M}=$ Longevity Adult Mosquito

$T_{E}=$ Longevity Infected Mosquito

$T_{I}=$ Longevity Infectious Mosquito

$I_{H}=$ Human Infectivity

$I_{R}=$ Mosquito Infectious Rate

\section{Figure 5}

contains a list of indicators with the abbreviations that we will use for the equations

MOSQUITO

INFECTIOUS RATE

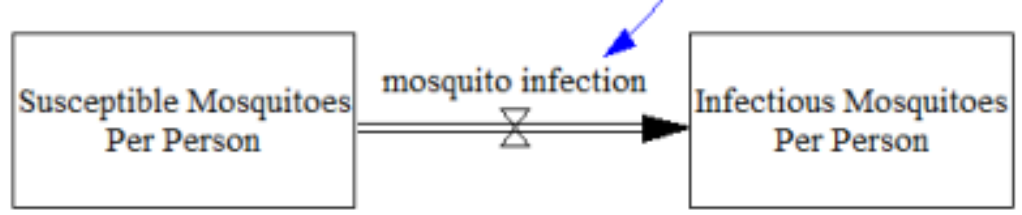

Figure 6

this simple version of the model we do not include the stock of infected mosquitoes so we connect directly susceptible with infectious through a flow of mosquitoes which is regulated by the infectious rate

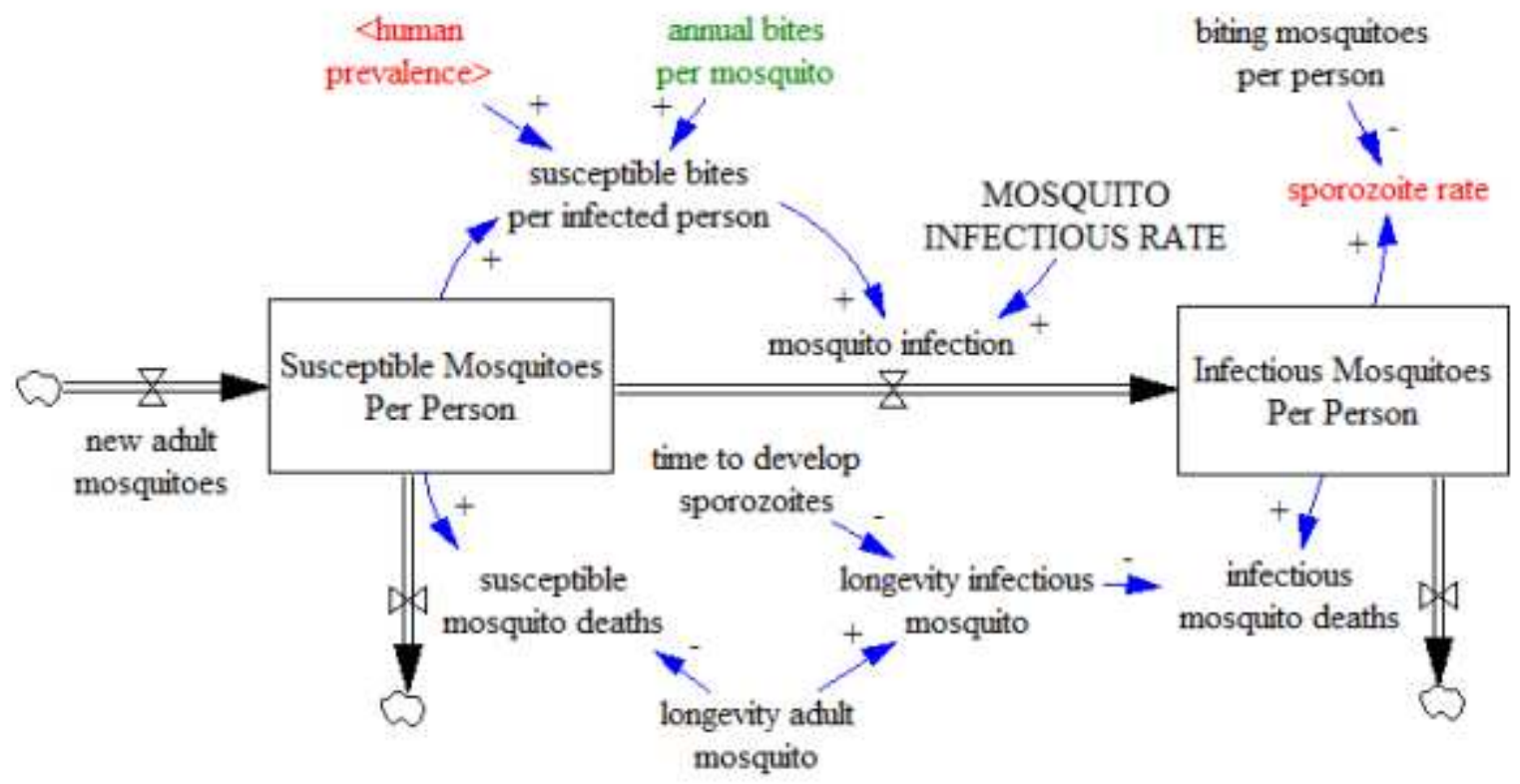


Figure 7

After dealing with the previous considerations, and once defined all the indicators of the mosquito sector, we can see them linked in the next model diagram

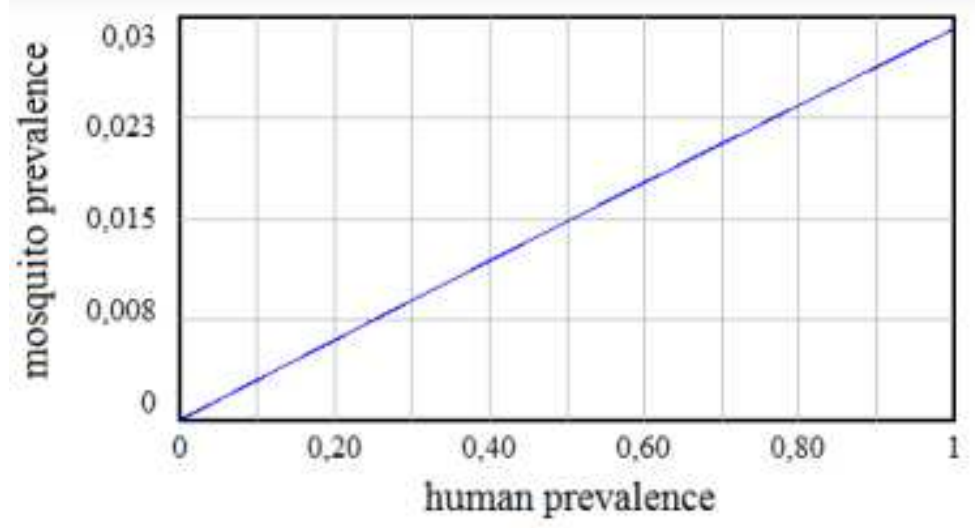

\section{Figure 8}

the maximum mosquito prevalence would be $2.9 \%$, which is reached when the area of study becomes holoendemic

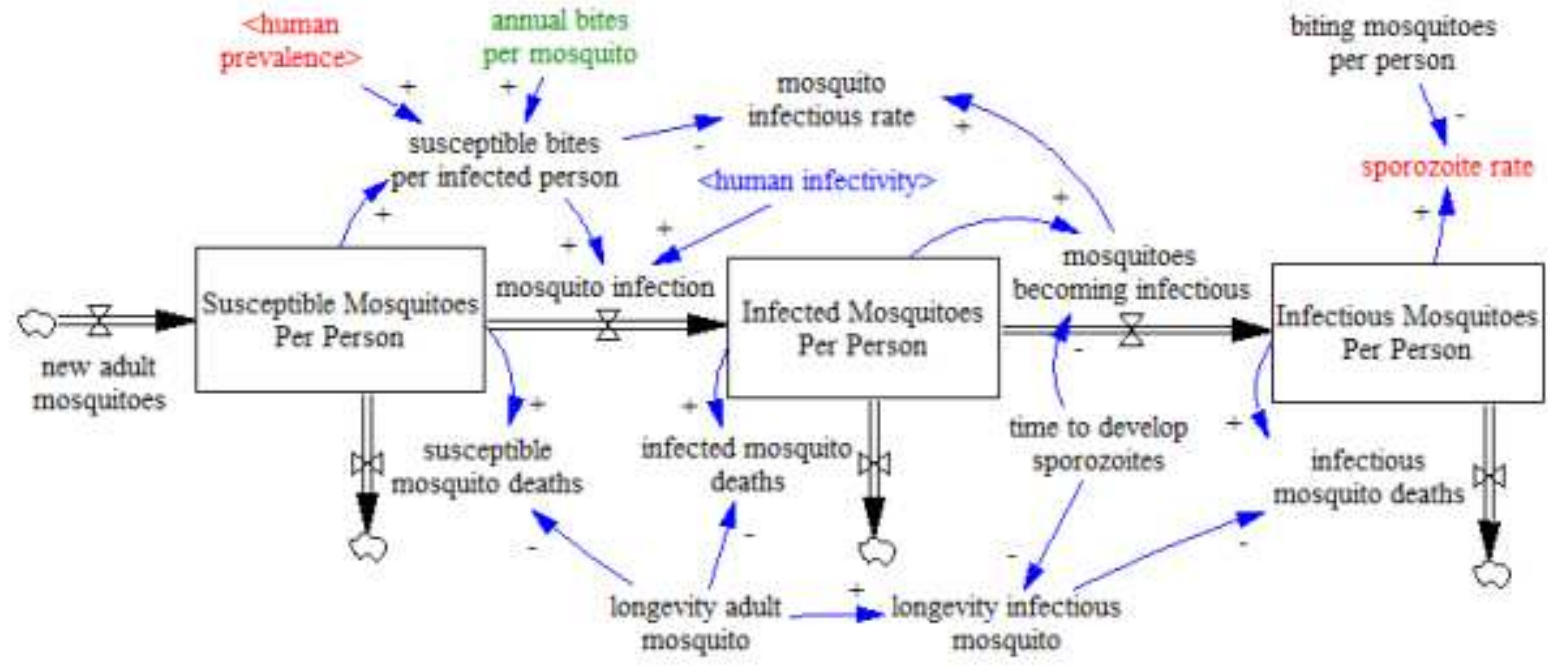

\section{Figure 9}

The new diagram includes the transitory stock of infected mosquitoes per person 


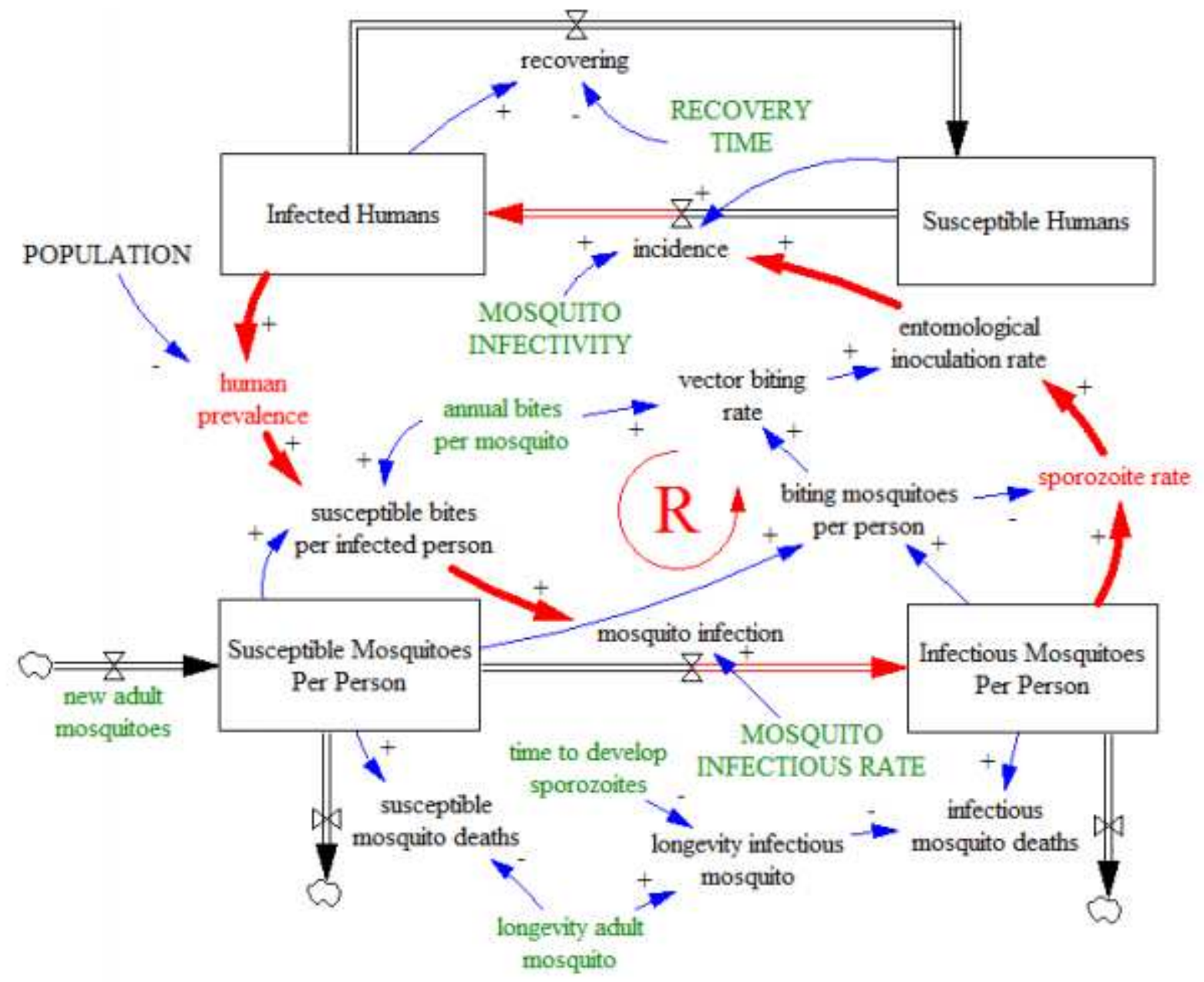

Figure 10

This major feedback loop is represented with red arrows

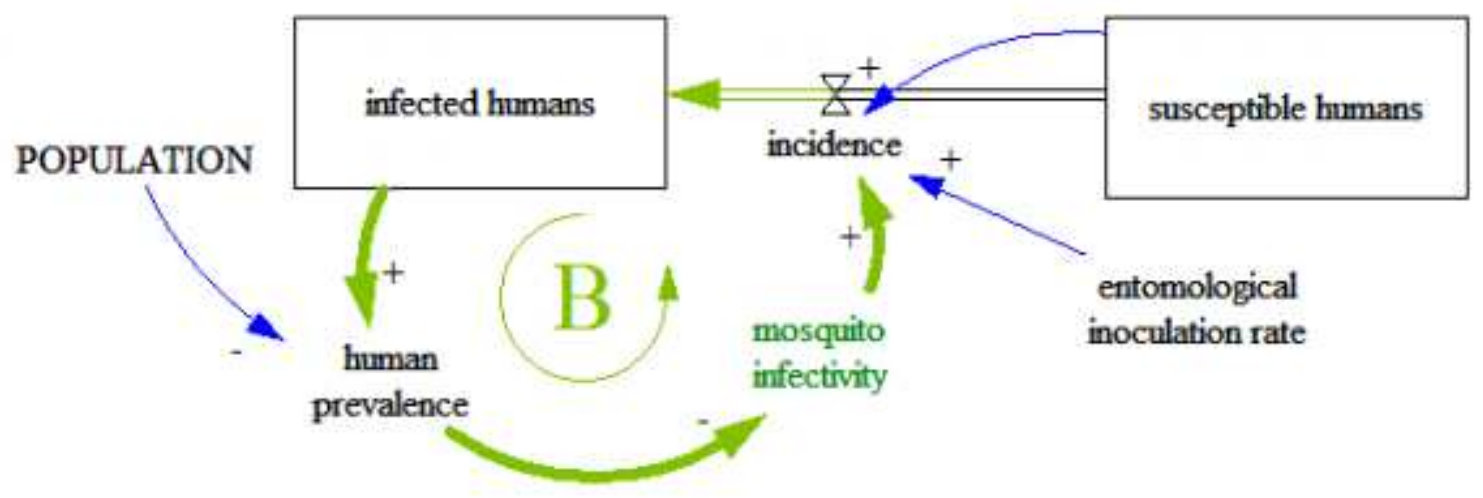

\section{Figure 11}

In this case, incidence would become smaller, which in return would reduce prevalence, generating a balancing loop a 